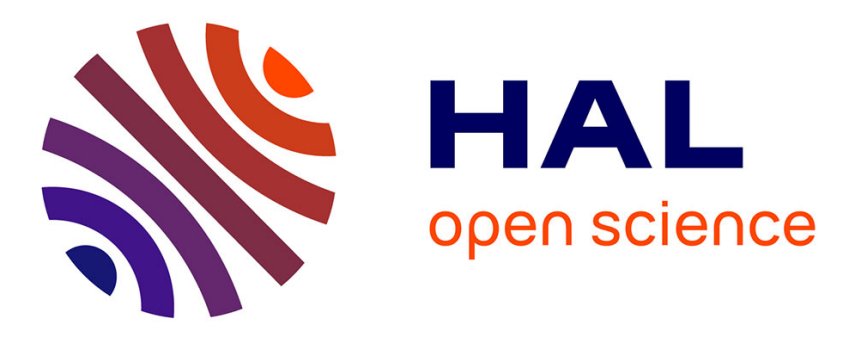

\title{
GATA1 pathogenic variants disrupt MYH10 silencing during megakaryopoiesis
}

Paul Saultier, Sandrine Cabantous, Michel Puceat, Franck Peiretti, Timothée Bigot, Noémie Saut, Jean-claude Bordet, Matthias Canault, Johannes Agthoven, Marie Loosveld, et al.

\section{To cite this version:}

Paul Saultier, Sandrine Cabantous, Michel Puceat, Franck Peiretti, Timothée Bigot, et al.. GATA1 pathogenic variants disrupt MYH10 silencing during megakaryopoiesis. Journal of Thrombosis and Haemostasis, 2021, 19 (9), pp.2287-2301. 10.1111/jth.15412 . hal-03374701

\section{HAL Id: hal-03374701 https://hal-amu.archives-ouvertes.fr/hal-03374701}

Submitted on 22 Oct 2021

HAL is a multi-disciplinary open access archive for the deposit and dissemination of scientific research documents, whether they are published or not. The documents may come from teaching and research institutions in France or abroad, or from public or private research centers.
L'archive ouverte pluridisciplinaire HAL, est destinée au dépôt et à la diffusion de documents scientifiques de niveau recherche, publiés ou non, émanant des établissements d'enseignement et de recherche français ou étrangers, des laboratoires publics ou privés. 


\section{GATA1 pathogenic variants disrupt MYH10 silencing during}

2 megakaryopoiesis.

3 Paul Saultier, ${ }^{1,2}$ Sandrine Cabantous, ${ }^{1}$ Michel Puceat, ${ }^{3}$ Franck Peiretti, ${ }^{1}$ Bigot Timothée, ${ }^{1}$ Noémie

4 Saut, ${ }^{1,4}$ Jean-Claude Bordet, ${ }^{5}$ Matthias Canault, ${ }^{1}$ Johannes van Agthoven, ${ }^{6}$ Marie Loosveld,,${ }^{4,7}$

5 Dominique Payet-Bornet ${ }^{7}$, Delphine Potier$^{7}$, Céline Falaise, ${ }^{2,4}$ Denis Bernot, ${ }^{1}$ Pierre Emmanuel

6 Morange,,$^{1,4}$ Marie-Christine Alessi, ${ }^{1,4^{*}}$ and Marjorie Poggi ${ }^{1}$

$7 \quad{ }^{1}$ Aix Marseille Univ, INSERM, INRAe, C2VN, Marseille, France

$8 \quad{ }^{2}$ APHM, La Timone Children's Hospital, Department of pediatric hematology, immunology and

9 oncology, Marseille, France

$10 \quad{ }^{3}$ Aix Marseille Univ, INSERM, MMG, Marseille, France

$11{ }^{4} \mathrm{APHM}, \mathrm{CHU}$ Timone, French Reference Center on Inherited Platelet Disorders, Marseille, France

12 5Unité d'Hémostase Biologique, Bron, France

13 'Structural Biology Program, Division of Nephrology/Department of Medicine, Massachusetts

14 General Hospital and Harvard Medical School, Charlestown, MA 02129, USA

$15 \quad{ }^{7}$ Aix-Marseille Univ, CNRS, INSERM, CIML, Marseille, France.

16

$17{ }^{*}$ Corresponding author: Marie-Christine Alessi, C2VN, Faculté de Médecine Timone, 27 Boulevard

18 Jean Moulin, 13385 Marseille, France ; E-mail : marie-christine.alessi@univ-amu.fr ; Tel : +33 491

19324507 ; Fax : +33 491254336.

20

21 Abstract: 238 words

22 Text: $\mathbf{5 0 0 2}$ words

23 Figures: 5

24 Tables: 3

25 Supporting Information: 9 figures and 2 tables

26 References: 41 
27 Abstract

28 Background

GATA1 is an essential transcription factor for both polyploidization and megakaryocyte (MK) differentiation. The polyploidization defect observed in GATA1 variant carriers is not well understood.

\section{Objective}

32 To extensively phenotype two pedigrees displaying different variants in the GATA1 gene and determine if GATA1 controls MYH10 expression levels, a key modulator of MK polyploidization.

34

\section{Method}

146 unrelated propositi with constitutional thrombocytopenia were screened on a multigene panel. We described the genotype-phenotype correlation in GATA1 variant carriers and investigated the effect of these novel variants on MYH10 transcription using luciferase constructs.

\section{Results}

The clinical profile associated with the p.L268M variant localized in the $C$ terminal Zinc finger was unusual in that the patient displayed bleeding and severe platelet aggregation defects without earlyonset thrombocytopenia. p.N206I localized in the $\mathrm{N}$ terminal Zinc finger was associated, on the other hand, with severe thrombocytopenia (15G/L) in early life. High MYH10 levels were evidenced in platelets of GATA1 variant carriers. Analysis of MKs anti-GATA1 ChIP-sequencing data revealed two GATA1 binding sites, located in the $3^{\prime}$ untranslated region and in intron 8 of the MYH1O gene. Luciferase reporter assays showed their respective role in the regulation of $\mathrm{MYH10}$ gene expression. Both GATA1 variants significantly alter intron 8 driven MYH10 transcription.

\section{Conclusion}

The discovery of an association between MYH10 and GATA1 is a novel one. Overall, this study suggests that impaired $\mathrm{MYH10}$ silencing via an intronic regulatory element is the most likely cause of GATA1-related polyploidization defect.

\section{Key words}

GATA1, MYH10, downstream targets, functional variants, platelets.

\section{Essentials}

- $\mathrm{MYH10}$ silencing is an essential step in the megakaryocyte polyploidization.

- Two novel GATA1 variants were identified in the $\mathrm{N}$ and $\mathrm{C}$-terminal zinc finger domain.

- MYH10 expression levels are increased in platelets from both GATA1 variant carriers.

- GATA1 alters MYH10 expression via an intronic binding site in the MYH10 gene. 
60

61

62

63

64

65

66

67

68

69

70

71

72

73

74

75

76

77

78

79

80

81

82

83

84

85

86

\section{Main Text}

\section{Introduction}

GATA1 is an essential transcription factor for erythrocyte and megakaryocyte (MK) differentiation $[1,2]$. It belongs to the GATA family of zinc finger (ZF) transcription factors, which recognize the $\mathrm{W}(\mathrm{A} / \mathrm{T}) \mathrm{GATAR}(\mathrm{A} / \mathrm{G})$ DNA pattern. The protein is encoded by the GATA1 gene, located on the $\mathrm{X}$ chromosome $[3,4]$. The GATA1 protein contains two highly conserved ZFs, which are separated by a short linker. The C-terminal ZF binds with high affinity and specificity to (A/T)GATA(A/G) motifs throughout the genome, while the N-terminal ZF can bind protein cofactors, such as FOG1.

Several hemizygous loss-of-function GATA1 variants have been described in patients with varying degrees of macrothrombocytopenia and anemia. Most of these variants affect the N-terminal ZF of GATA1 and impair the GATA1 DNA binding interaction with the GATA1 cofactor FOG1 [5-7]. Accordingly, mice with targeted mutations in the Gata1 promoter display macrothrombocytopenia, in which reduced Gata1 expression [8-10] or conditional knockout of Gata1 results in a selective loss of Gata1 expression in MKs [11]. This observation underscores the importance of GATA1 in sustaining normal megakaryocytic differentiation and platelet production. Previous studies have shown that GATA1 is required for both polyploidization and MK differentiation. Bone marrow from GATA1 variant carriers shows a moderate to marked increase in MK numbers, which are also small and hypolobulated $[5,6] . I n$ vivo, the Gata1 ${ }^{\text {low }}$ mice display a marked increase in small and hypoploid MKs [9]. GATA1-deficient MKs also exhibit increased proliferation both in vitro and in vivo with defective nuclear and cytoplasmic maturation $[9,10]$.

Some studies have suggested that the polyploidization defect is associated with GATA1 inactivation through deregulation of cyclin D1, STAT1 or CDC6 expression [10,12]. However, we propose an alternative mechanism involving altered silencing of the protein non-muscle heavy chain IIB (MYH10). During polyploidization, expression of MYH10 protein, which localizes to the actomyosin contractile ring, is repressed by the transcription repressor RUNX1, thereby enabling the switch from mitosis to endomitosis [13]. The polyploidization defect observed in RUNX1 variant carriers is in part associated with the persistence of MYH10 expression [14], which has been detected in circulating platelets [15]. 
87 Similarly, increased levels of MYH10 protein have been detected in platelets from patients carrying

88 FLI1 variants, thus suggesting cooperation between the two transcription factors, RUNX1 and FLI1, 89 in normal repression of the MYH10 gene [15-18].

90 Genome-wide analysis of simultaneous GATA1, RUNX1, FLI1 and TAL1 DNA binding in human MKs 91 has revealed that these transcription factors bind to the same binding sites in the genome and 92 cooperate to regulate the expression of target genes during the early stages of megakaryopoiesis 93 [19]. Transcriptional cooperation between RUNX1 and GATA1 in the activation of megakaryocytic 94 promoters has been further established using reporter assays with transient transfection in a variety 95 of mammalian cell lines. These studies have demonstrated the physical interaction between the two 96 transcription factors [20,21]. Therefore, we suggest that deregulation of $M Y H 10$ expression may also 97 be implicated in GATA1-related thrombocytopenia.

98 In this study, we report a detailed analysis of two novel GATA1 variants localized in each ZF. Elevated 99 MYH10 protein levels were found in platelets from carriers of each variant. We describe the 100 identification of a MYH10 intronic region that functions as a GATA1-responsive cis-regulatory 101 element.

102 


\section{Materials and Methods}

104 Methods concerning high-throughput gene sequencing, structural model of GATA1-DNA interactions,

105 flow cytometry analysis, cell expression and luciferase reporter assays, platelet-rich plasma (PRP) serotonin level, platelet PAI-1 level, mepacrine uptake and release, immunoprecipitation and western blot analysis, and epifluorescence microscopy are described in the Online Supplementary Material.

108 Study limitations: Owing to the limited number of patients, statistical comparison between patients 109 and controls was not always possible. Fresh whole blood (required for primary cell culture) was 110 available in only one patient (F1-II1). Consequently, most in vitro experiment was performed using 111 transfected non-hematopoietic cell line. The platelet serotonin levels were available for two patients 112 and the BM smears for one patient. TEM analysis of platelets were performed for only one family. 113 The others experiments were performed for all the patients.

\section{Patient enrollment, research ethics, patient consent, and clinical and laboratory data}

115 From 2014 to 2017, we recruited 146 unrelated propositi with constitutional thrombocytopenia of 116 unknown origin. Genetic analyses were performed at the French Reference Center for Inherited 117 Platelet Disorders at La Timone University Hospital in Marseille, France. All cases were included in 118 the study after obtaining informed written consent in accordance with protocols approved by national 119 institutional review boards and the Declaration of Helsinki principles. Medical and family history data 120 were obtained from medical reports and patient interviews. Bleeding tendency was assessed using 121 the International Society on Hemostasis and Thrombosis-Bleeding Assessment Tool (ISTH-BAT) 122 score. Red blood cell counts, platelet counts, mean corpuscular volume (MCV) and mean platelet 123 volume (MPV) were determined on EDTA-anticoagulated blood samples using the ADVIA 120 124 automated cell counter. Bone marrow smears were obtained for one propositus and stained with May125 Grünwald-Giemsa. Fetal hemoglobin levels were assessed using high-performance liquid 126 chromatography in the Biochemistry Department at La Timone University Hospital in Marseille, 127 France. 
128 Platelet aggregation

129 Platelet aggregation was assessed by measuring light transmission through the stirred platelet-rich 130 plasma (PRP) suspensions for 3 min using an APACT 4004 aggregometer. Platelet aggregation was 131 triggered by adding $1 \mu \mathrm{M}$ or $2.5 \mu \mathrm{M}$ adenosine diphosphate (ADP; Helena laboratories), $0.5 \mathrm{mg} / \mathrm{ml}$ 132 arachidonic acid (Helena laboratories) and $0.36 \mu \mathrm{g} / \mathrm{ml}$ or $0.72 \mu \mathrm{g} / \mathrm{ml}$ collagen (Bio/Data Corporation). 133 Platelet agglutination was triggered by adding $1.5 \mathrm{mg} / \mathrm{ml}$ ristocetin (Sigma-Aldrich).

\section{Evaluation of platelet granules using electron microscopy}

135 Transmission electron microscopy (TEM) analysis of platelets was performed as previously described 136 [18]. Briefly, we assessed platelet surface area $\left(\mu \mathrm{m}^{2}\right)$ and diameter $(\mu \mathrm{m})$, the number of alpha 137 granules per unit area $\left(\mu \mathrm{m}^{-2}\right)$, and the diameter of alpha granules in a blinded case/control 138 experiment. Dense granules were quantified using whole-mount electron microscopy as previously 139 described [18].

140 In vitro megakaryocyte differentiation and ploidy

141 After density gradient separation (Eurobio), circulating $\mathrm{CD}_{3} 4^{+}$cells were purified using positive 142 selection with magnetic beads (Miltenyi-Biotec) and cultured in StemSpan Serum-Free Expansion 143 Medium II supplemented with Megakaryocyte Expansion Supplement (Stemcell Technologies). At 144 days 9 and 11 of culture, MKs were analyzed via flow cytometry for CD41 and CD42a markers (anti145 CD41-APC and anti-CD42a-PE antibodies, BD Biosciences) as well as for DNA ploidy using Hoechst14633342 (Sigma-Aldrich). The cells were then analyzed using a Navios cytometer (Beckman Coulter).

\section{Statistical analyses}

148 Quantitative variables are expressed as the mean \pm standard error. Analyses were performed using 149 GraphPad Prism software. Statistical differences were determined via ANOVA with Dunnett's multiple 150 comparison test. $\mathrm{P}<0.05$ was considered statistically significant. 
152 Results

153 Two novel GATA1 variants identified via genetic analysis

154 Next-generation sequencing yielded the identification of two novel GATA1 variants (NM_002049) in 155 three boys from two unrelated families (Figures 1A-B). For the first family, only one hemizygous 156 variant c.617A $>\mathrm{T}$ (p.N206I) causing alteration in the N-terminal ZF was evidenced in the proband (II157 1) (Fig 1A). For the second family only one deleterious variant c.802C>A (p. L268M) was shared by 158 the two brothers in the C terminal ZF GATA1 domain. Five other variants worn by the two brothers 159 have been identified. None of them were pathogenic due to benign in silico prediction and/or high 160 allelic frequency $\left(>10^{-4}\right)$ in general population databases (Supplemental Table 1). The analysis of the 161 coverage of these genes was also not in favor of deletion or duplication (data not shown). These two 162 missense variants were absent from the GnomAD and ExAC databases, and have a Combined 163 Annotation Dependent Depletion (CADD) score of 24.9 for c.617A>T (Request: Chromosome X, 164 Position 48792341, CADD GRCh38-v1.6) and 23.7 for c.802C>A (Request: Chromosome X, Position 48793229, CADD GRCh38-v1.6). The chart prediction for these two variants based on different

166 prediction tools to identify disease relevant non-synonymous single nucleotide variants is given in 167 supplementary figure (Supplemental Figure $1 \mathrm{~A}$ and $\mathrm{B}$ ). The variants occurred in both mothers, while 168 as expected, the two fathers were homozygous for wild type genotypes (Figure 1A).

169 Structural modeling

170 Structural analysis provided further insight into the mechanisms by which these two variants may 171 exert deleterious effects. An initial sequence alignment of GATA1 orthologs and paralogs revealed 172 that both variants affect highly conserved sequences and may therefore play a structural role in 173 GATA1 binding to target DNA sequences or protein binding partners (Supplemental Figure 1). As 174 shown in the X-ray structure of GATA1 in complex with double-stranded DNA, the L268 residue is 175 located in the interface between GATA1 and the major groove of the DNA-binding sequence TGATAA 176 (Figure 1D, left), making van der Waals contact with bases 32T, 33T, 9T and 10C (distance cutoff of $1774 \AA$ ). Structural modeling of the p.L268M substitution did not reveal any steric hindrance at the GATA1- 
DNA interface. However, the loss-of-function effect of p.L268M likely results from distorted hydrophobic packing with the aforementioned bases (shown in the left inset of Figure 1D). Although the N206 residue is not localized within the GATA1-DNA interface (Figure 1D, left), the p.N206I substitution may affect the capacity of GATA1 to bind to FOG1. The nuclear magnetic resonance structures of the GATA1-FOG1 complex revealed that the N206 residue is part of the interface between the two proteins, forming a specific amino-m interaction between the GATA1 side chain amino group and the aromatic ring of FOG1-Y30 (Figure 1D, right). Structural modeling showed that this interaction is disrupted in the N2016I variant. The variant caused destabilization of the GATA1/FOG1 interface by steric hindrance, likely resulting in FOG1-mediated loss of function (shown in the right inset of Figure 1D).

\section{Nuclear localization and FOG1 binding in GATA1 cases}

To further investigate the subcellular localization of the GATA1 variants, we expressed the wild-type and variant forms of GATA1 in MSR and H9C2 cells. GATA1 variants did not reduced GATA1 protein levels (data not shown). The GATA1 variants entered the nucleus to the same extent as wild-type GATA1 (Supplemental Figure 2A), thereby suggesting that the variants alter GATA1 binding properties to chromatin rather than subcellular localization. The structural prediction was confirmed via GATA1 co-immunoprecipitation using MSR cells overexpressing GATA1 (WT or variants) and FOG1. FOG1 was detected in the GATA1 precipitates of wild-type and p.L268M GATA1-expressing cells, while FOG1 detection was markedly reduced in the p.N206I GATA1 precipitates (Supplemental 197 Figure 2B).

\section{Case descriptions}

199 Thrombocytopenia onset kinetics markedly differed between the two families. Thrombocytopenia was 200 present early in life in the p.N206I carrier (F1-II-1) with a severely reduced platelet count (15 G/L) at 201 the age of 18 months that persisted. Bleeding during dental avulsion was prevented by platelet 202 concentrate transfusion prior to the procedure. The patient was referred to our reference center on 203 platelet disorders because of resistance to immunoglobulin, corticosteroids and mycophenolate 204 mofetil treatment, as immune thrombocytopenia was initially suspected. The F1 proband did not suffer 
205 from anemia (hemoglobin $118 \mathrm{~g} / \mathrm{L}$ at seven years of age), and his mean corpuscular volume was 206 rather low (76.8 fL- normal range: 80-95 fL). May-Grunwald-Giemsa staining of bone marrow smears 207 showed mild dyserythropoiesis with erythroblastosis and nuclear karyorrhexis (Figure 2C).

208 By contrast, thrombocytopenia was initially absent in the two F2 brothers carrying the p.L268M 209 variant. At 18 and 9 months of age, F2-II-1 and F2-II-2 exhibited normal platelet counts (261 G/L and $210233 \mathrm{G} / \mathrm{L})$, respectively despite pronounced mucocutaneous bleedings. The initial hemostasis 211 assessment in the two children showed no defects in coagulation (normal prothrombin and activated 212 partial thromboplastin times, normal levels of the factors VIII, XIII, fibrinogen and von Willebrand 213 factor) or fibrinolysis (normal alpha2 antiplasmin, t-PA and plasma PAI-1 levels and normal von Kaulla 214 test) (data not shown). Bleeding time was prolonged (>15 $\mathrm{min}$ ) and was not corrected by DDAVP 215 injection. A severe platelet aggregation defect was detected in both brothers with normal platelet 216 counts in PRP samples (338-380G/L). On several occasions (6 times for the older brother and 3 times 217 for the younger brother) and in the absence of medication, collagen, arachidonic acid and TXA2 218 agonist U-46619 failed to induce platelet aggregation. Reduced platelet aggregation was also 219 observed in response to ADP, while ristocetin-induced agglutination was almost normal (Table 1 and 220 Supplemental Figure 3). Both boys were diagnosed with hereditary platelet dysfunction. Diagnosis of 221 Glanzmann thrombasthenia has been evoked but platelet exploration has refuted it. Indeed, platelet 222 aggregation was only slightly reduced upon stimulation with high doses ADP (10 $\mu \mathrm{M})$ and TRAP-14 $223(50 \mu \mathrm{M})$. Exploration of GPIIbIlla with flow cytometry showed a normal expression level of GPIIbIlla 224 (Table 2) and a significant increment of PAC-1 fixation after $10 \mu \mathrm{M}$ ADP stimulation (MFI: basal state= 2250.31 (normal value 0.21-0.49) stimulated state $=6.23$ (normal value: $5.4-16.2$ ), positive cell: basal 226 state $=0 \%$ (normal value $<5 \%$ ), stimulated state $=73 \%$ (normal value $>70 \%$ )). In addition, we did not 227 detect variations, deletion or duplication in the ITGA2B and ITGB3 genes and in genes involved in its 228 activation (FERMT3, RASGRP2, TLN1). The altered aggregation profiles was not due to defective 229 expression of GPVI, SYK, PLCY2 or COX1 in platelets, with no major differences in signal intensity 230 between the two parents and the children. A 30\% reduction in TBXAS1 levels was obseverd in both 231 F2 children compared to their parents (Supplemental Figure 4). In patient F2-II-2, dental avulsions 
and an appendectomy were performed after platelet concentrate transfusions without hemorrhagic complications. At five years of age, the platelet counts for patient F2-II-1 remained in the normal range $\left(194 \times 10^{9} / L\right)$, while his brother's platelet counts started to decrease at three years of age $(131 \times$ $\left.10^{9} / \mathrm{L}\right)$. Thrombocytopenia then gradually developed over time in both brothers, with more severe thrombocytopenia in the younger boy (F2-II-2) (Figure 2A). TEM analysis of platelets derived from patient F2-II-1 showed that, in keeping with previous measurements of mean platelet volume obtained using hematology analyzers, the platelet size distribution was normal (Figure 3B and Supplemental Table).

As GATA1 is critical for erythropoiesis, we also aimed to identify any red blood cell defects. Examination of peripheral F2-II-2 blood smears demonstrated abnormally shaped red blood cells with anisocytosis and poikilocytosis (Figure 2B). The F2 probands did not suffer from anemia either, with hemoglobin levels at 111 and $112 \mathrm{~g} / \mathrm{L}$ at five years of age and 146 and $144 \mathrm{~g} / \mathrm{L}$ at the date of last follow-up (at 27 and 23 years of age, respectively). Hemoglobin electrophoresis revealed, as already reported in several GATA1 related diseases, an elevated level of fetal hemoglobin. They both presented with elevated fetal hemoglobin levels (25\%), albeit without microcytosis (MCV: 87-92 fL between five and eight years of age) and hypochromia (mean corpuscular hemoglobin concentration: 330-331 g/L between five and eight years of age). Remarkably, the two F2 brothers displayed a progressive increase in MCV over time, while their platelet counts gradually decreased (Figure 2A); the values at last follow-up were 99 and $103 \mathrm{fL}$ (at 26 and 23 years of age, respectively). Table 3 resumes main characteristics of GATA1 variant carriers.

\section{Platelets from GATA1 cases present severe dense granular defect}

Electron microscopy revealed a major delta granular deficit in GATA1 variant carriers. Analysis via whole-mount electron microscopy revealed reduced numbers of dense granules, with an average of $0.44 \pm 1.02$ (F1-II-1), $0.56 \pm 0.97(\mathrm{~F} 2-\mathrm{II}-1)$ and $0.13 \pm 0.49$ (F2-II-2) dense granules per platelet lacked dense granules (Figure 3A). Mepacrine uptake and release upon TRAP-14 stimulation (40 $\mu \mathrm{M})$ and quantification of mepacrine-labeled dense granules via fluorescent microscopy confirmed 
the dense granule defect in all variant carriers (Supplemental Figure 5). On several occasions, we observed low platelet serotonin levels for the two F2 brothers (F2-II-1: $0.12 \mu \mathrm{g} / 109$ platelets in 2015, $0.10 \mu \mathrm{g} / 109$ platelets in 2009 and F2-II-2: $0.16 \mu \mathrm{g} / 109$ platelets in 2015, normal range: 0.3-1.2 $\mu \mathrm{g} / 109$ platelets). Flow cytometry showed reduced expression levels of the dense granule marker CD63 upon TRAP-14 stimulation in all variant carriers (Table 2). Platelet count (Table 1), platelet aggregation (Supplemental Figure 3), flow cytometric evaluation of mepacrine uptake and release (not shown) and platelet serotonin levels $(0.39 \mu \mathrm{g} / 109$ platelets, normal range: $0.3-1.20 \mu \mathrm{g} / 109$ platelets) were normal in the mother (F2-I-1).

Platelets from GATA1 cases present mild alpha granular defect and structural abnormalities

Assessment of peripheral blood smears from F1-II-1 and F2-II-2 did not show gray platelets, although an experienced hematologist identified some hypogranular platelets in both children (Figure 2B).

271 Quantitative morphometric evaluation of F2-II-1 alpha granules indicated a distinct decrease in alpha 272 granule density and consequently a decrease in the alpha granule area: total platelet area ratio (4.1\%, 273 reference range $11.0-13.5 \%)$. The diameter of the alpha granules was only slightly decreased. The 274 platelets presented many other structural anomalies. Platelet sections showed highly variable 275 granular and mitochondrial content, with large areas of membrane complexes that in some cases occupied the entire section (See Figure 3B legend for complete description). To further assess the 277 alpha granules, we evaluated the levels of PAl-1 antigen in serum. Indeed, 95\% of circulating PAI-1 278 is localized in platelets 4. Furthermore, proteomic analysis has found that reduced PAl-1 levels is a 279 feature of gray platelet syndrome5. For the three patients, PAI-1 levels were in the normal range (F1280 II-1: 0.35, F2-II-1: 0.76 and F2-II-2: 0.69, normal range 0.33-1.07 ng/106 platelets). Flow cytometry 281 showed moderate decrease in the levels of the alpha granule protein marker CD62P upon TRAP-14 282 stimulation in both the F2 children's whereas CD62P did not change after TRAP-14 stimulation in the 283 F1-II-1 (Table 2). 
GATA1 variants are associated with abnormal megakaryocyte maturation and polyploidization.

Microscopic evaluation of bone marrow aspirate smears from patient F1-II-1 showed normal numbers of MKs with a high proportion of round or hypolobulated polyploid nuclei (Figure 2C) indicating abnormal maturation. We further analyzed MK development using blood-derived CD34+ stem cells. The F1 carriers exhibited a 12 - to 48 -fold increase in circulating $C D 34^{+} C D 38^{+} C D 45^{\text {low } C D 19 \text { low }}$ cells $(n=2)$ compared with healthy controls $(n=5)$ (Figure 4A). Analysis of CD41+ and CD42a ${ }^{+}$cells at days 9 and 11 revealed a marked reduction in MK differentiation with reduced cell size compared with two controls (Figures 4B-C). The CD41+CD42a ${ }^{+}$population was almost absent in the F1-II-1 cells (Figure 4B). Furthermore, the percentage of high-ploidy cells $(\geq 4 n)$ in the F1-II-1 sample was reduced at day 9 . The relative frequencies of $2 n, 4 n, 8 n$ and $16 n$ ploidy MKs were $54-58 \%, 23-27 \%, 4-8 \%$ and $1-2 \%$ in the controls, versus $66 \%, 18 \%, 0 \%$ and $0 \%$ in the patient carrying the GATA1 variant, respectively (Figure 4D). Bone marrow smears and MK cultures were not available for the F2 family. GATA1 cases display increased MYH10 levels in platelets

To further investigate the effect of the GATA1 variants on MYH10, which is required for MK polyploidization and maturation, MYH10 levels were assessed in platelets derived from patients and

301 healthy subjects. MYH10 protein was not detected in healthy donor platelets via immunoblotting. By

302 contrast, MYH10 expression was detected in platelets from the three probands and one carrier of a 303 FLI1 variant as previously reported [18]. MYH10 protein was also present in the mother of the F2 304 probands (F2-I-1), albeit at relatively reduced levels (Figure 4E). The polyploidization defect has been 305 shown associated through deregulation of cyclin D1-Cdk4 or CDC6 expression [10,12]. In MSR cells, 306 GATA1 wild-type or mutants' transfection did not altered their expression levels (Supplemental figure $3076)$.

\section{Evidence of a $\mathrm{MYH10}$ intronic regulatory region}

309 Our data suggests that GATA1 regulates MYH10 expression. Therefore, we obtained GATA1, 310 RUNX1, and FLI1 ChIP-sequence data on primary human MKs (deposited by the Göttgens 311 laboratory) from the Gene Expression Omnibus (GEO) database [19]. The peak enrichments on 
312 chromosome 17, which includes the MYH10 locus, were extracted and displayed in the UCSC 313 genome browser. This analysis showed two enriched binding regions for the GATA1, RUNX1, and 314 FLI1 transcription factors in the MYH10 gene (displayed from ENCODE data using the 315 erythroleukemia K562 cell line known to express GATA1) (Figure 5A).

316 The first binding peak (Chr. 17: 8472947-8473157) located in the MYH10 3'UTR corresponded to 317 GATA1 and RUNX1 binding sites. Remarkably, sequence analysis did not retrieve the consensus 318 GATA1 binding motif in this region. The second peak (Chr. 17: 8553047-8553739) was located within 319 an intronic region and contained potential binding sites for GATA1, FLI1 and RUNX1. Four potential 320 GATA1 binding sites (referred to as BS-1, BS-2, BS-3 and BS-4) were identified, of which three 321 contain the reverted sequence TATC (BS-2, BS-3 and BS-4) (Supplemental Figure 7). We also 322 uploaded the chromatin interaction analysis with paired-end tag sequencing data for CTCF and POL2 323 performed on the K562 cell line from ENCODE, using the UCSC genome browser. This analysis 324 revealed that the intronic enhancer was indeed not occupied by Pol2 or CTCF (data not shown). The 325 interaction between gene regulatory regions (displayed with data from ENCODE) revealed that the 326 intronic site was likely within a close chromatin region in a DNA repressive loop between two CTCF 327 enriched regions (data not shown).

\section{Transcriptional activity of the MYH10 intronic element}

329 We compared the 3'UTR and intronic regulatory element-driven expression of MYH10 using a 330 luciferase reporter assay in two cell lines expressing or not the GATA1 hematopoietic partners. The 331 HEL cell line expresses the GATA1, RUNX1 and FLI1 transcription factors. In HEL cells, both the 332 3'UTR and intronic regions significantly repressed luciferase expression compared with the pGL3 333 empty vector (control) (Figure 5B). Removal of only one of the four GATA1 binding sites in MYH10 334 intron 8 region was sufficient to yield markedly reduced intronic activity.

335 We then look at the effect of GATA1 variant on intron 8 driven MYH10 expression. Since HEL cells 336 express endogenous GATA1 we used a non-hematopoietic model, the MSR epithelial cells which do 337 not express basal levels of GATA1, RUNX1 and FLI1 (Supplemental Figure 7). In this model wild338 type GATA1 transfection enhanced luciferase activity via the MYH10 intronic region (Figure 5C left), 
but not via the 3'UTR region (Figure 5C right). Disruption of all four GATA binding sites in the intronic region yielded a significant reduction in luciferase activity (approximately 40\%). Disruption of BS-2 (M2) or BS-4 (M4) also led to a significant reduction in luciferase activity, while disruption of BS-1

We then evaluated the variants effects in MSR cells. Both here reported (N206L and L268M GATA1) and already described (V205M and G208S) GATA1 variants were associated with a marked reduction in transcriptional activity compared with wild-type GATA1 (Figure 5D and supplemental figure 8).

\section{Discussion}

In this study, we assessed two pedigrees displaying a variant in the GATA1 gene. We report the first description of a variant in the C-terminal ZF of GATA1 that initially manifested as mucocutaneous bleeding with platelet dysfunction but without thrombocytopenia, which appeared later in life. We

352 during MK differentiation, which may explain the decrease in MK ploidy and reduced platelet

353 formation. Assessing published ChIP-sequence data and using reporter cell and mutagenesis 354 assays, we demonstrated that GATA1 repressed MYH10 expression via intronic binding sites in the 355 MYH10 gene.

356 To date, nearly all reported GATA1 variants have been shown to involve the N-terminal ZF (positions $357205,208,216$ and 218) and the intron/exon boundaries of exon 2 [22]; with the exception of the 358 terminal p.X414R variant, which cause removal of the terminal codon and adds 42 extra amino acids 359 to the full-length GATA1 protein [23]. Variants in the N-terminal ZF and at position 414 are associated 360 with thrombocytopenia, while variants in exon 2 essentially lead to changes in the red blood cell 361 lineage, leading to severe anemia or Diamond-Blackfan anemia. Currently, variants in the C-terminal 362 ZF have not been described. The p.H289R variant, localized immediately 3 ' of the C-terminal ZF, has 363 been reported to be not as damaging as variants affecting the N-terminal ZF domain [24].

364 In this study, we describe two novel GATA1 variants. The patient harboring the variant at position 206 365 suffered from moderate bleeding with severe thrombocytopenia and mild dyserythropoietic features. 
366 This observation agrees with previous descriptions of $\mathrm{N}$-terminal ZF variants. Accordingly, this 367 variation impairs FOG1 binding. The second variant identified affected the C-terminal ZF. Unlike 368 variations in the N-terminal ZF, the p.L268M variation does not affect the GATA1/FOG1 interaction. 369 The clinical characteristics resemble those observed with the p.R216Q variant localized in the DNA370 binding surface of the $\mathrm{N}$-terminal ZF, including macrothrombocytopenia, red blood cell abnormalities 371 and minor beta-thalassemia [25-28]. Presentation was also close to the one described for the H289R 372 variation. As in the F2 patients, the hemizygous males harbouring the H289R substitution exhibit 373 increased MCV but normal hemoglobin levels. Thrombocytopenia was present only in two of the four 374 affected family members [24]. Although both F2 children presented with high level of fetal hemoglobin, 375 the absence of thrombocytopenia did not initially direct towards a GATA1 related disease. Indeed, 376 the disease initially manifested as severe mucocutaneous bleeding associated with reduced platelet 377 aggregation without thrombocytopenia. Platelet exploration carried out on several occasions did not 378 make it possible to link the platelet phenotype to a known thrombopathy. Indeed, GPIIbIlla was

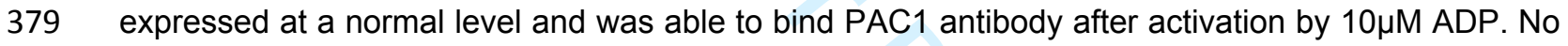
380 sign of immune deficiency was observed in these children excluding a kindlin 3 deficiency [29]. Finally, 381 the results of platelet aggregation were not in favor of a CaIDAG-GEFI deficiency as platelet activation 382 induced by a high concentration of arachidonic acid, identical to the one used in this article usually 383 gives normal results [30]. For a long time, these children were considered to be carriers of a storage 384 pool disease because of the early detection of a severe deficit of dense granules. These results are 385 to be connected with those of White et al [31] who observed a near absence of dense bodies in the 386 platelets from a patient with the R216Q GATA1 mutation. Although rescue experiments have not be 387 performed and causality has not been shown, our results support the idea that the spectrum of 388 GATA1-related diseases may be extended to include X-linked bleeding diathesis without 389 thrombocytopenia, associated with collagen and arachidonic acid aggregation defects. In contrast to 390 studies in Gata1-deficient mice [9,11,27,32] we did not observe reduced protein levels of GPVI, SYK 391 or other key molecules involved in collagen signaling pathways, such as PLCY2 that could explain 392 the aggregation defect. The decrease in TBXAS1 levels observed in the two F2 brothers may play a 
role in the reduced response to arachidonic acid. However, the platelets also failed to respond to U46619, thus indicating a more complex mechanism. The granule deficiency observed in the current study may also contribute to the platelet aggregation defects, although a complete lack of arachidonic acid- or collagen-induced platelet aggregation is not a feature of granule deficiency. Overall, further studies are required to understand the cause of platelet dysfunction observed in GATA1 variant carriers. Thrombocytopenia developed gradually over time associated with an enlargement in red blood cells, indicating progression of the disease in p.L268M carriers. Cell-type-specific gene regulation may change during ontogeny. There are substantial developmental differences between neonates and adults in the process of megakaryopoiesis and in their responses to TPO [33]. Wenqing

402 and colleagues have highlighted fundamental and conserved differences at distinct developmental 403 stages, characterized by simpler promoter-centric regulation of cell-type-specific genes in embryonic 404 cells and increased combinatorial enhancer-driven control in adult cells [34]. Overall, the delayed 405 onset of thrombocytopenia observed in the F2 family suggest developmental stage-specific GATA-1 406 regulation.

407 Assessment of MKs in GATA1 variant carriers revealed polyploidization and differentiation defects. 408 Assessment of the p.N206I variant revealed a marked decrease in mature CD41 high CD42ahigh MKs, 409 along with an increase in CD41-CD42a- cells. Although we cannot formally exclude impaired 410 expression of CD41 and CD42a in MKs harboring a GATA1 variant, the reduction in high-ploidy cells 411 confirmed the MK maturation defect.

412 Additionally, the GATA1 N206I carrier displayed an increase in circulating CD34+ progenitor cells, as 413 described for individuals carrying a germline ETV6 variants [27]. Recently, Shin et al. [36] have found 414 that Gata1 low megakaryocyte-erythroid progenitors/megakaryocyte precursors cell lines display 415 accelerated replication after approximately 90-100 days of culture. The observed increase in CD34+ 416 cells may reflect this high proliferative capacity.

417 The polyploidization defect associated with GATA1 is not well understood. MYH10 has been shown 418 to be required for cytokinesis during meiotic cell division [37] and to contribute to MK polyploidisation $419 \quad[13,38]$. RUNX1-mediated silencing of $M Y H 10$ has been found to be required for MK polyploidization 
420 and may serve as a biomarker of RUNX1 variants in patients [15]. FLI1 forms a complex with RUNX1

421 to regulate megakaryopoiesis. Carriers of FLI1 variants also exhibit elevated MYH10 levels in 422 platelets [15-17]. Interestingly, using a genome-wide analysis of simultaneous transcription factor 423 binding in human MKs and ENCODE ChIP-sequence data, we have shown that FLI1, RUNX1 and 424 GATA1 accumulate at regulatory elements in intron 8 and the 3'UTR of the MYH10 gene [19]. 425 Additionally, these transcription factors cooperate during megakaryopoiesis as components of a large 426 transcriptional complex [21,39], thereby suggesting that GATA1 may regulate MYH10 expression in 427 cooperation with RUNX1 and FLI1. To our knowledge, MYH10 has not yet been described as a target 428 gene of GATA1. We observed abnormal persistence of MYH10 protein in the platelets of the three 429 GATA1 variant carriers. The mother of the two F2 brothers also exhibited a slight increase in MYH10 430 levels. In a reporter luciferase assay using the HEL cell line, both regulatory elements in intron 8 and 431 the 3'UTR of MYH10 were found be functional by inducing reduced transcriptional activity. Disruption 432 of the four intronic binding sites fully reversed the repressive activity, thus indicating that the intronic 433 binding sites are required for MYH10 repression, despite the presence of the other repressive 434 complex partners (i.e., RUNX1, FLI1). The 3'UTR region of MYH10 may preferentially bind to a portion 435 of a protein complex that includes GATA1, without binding directly to GATA1. While GATA1 wild-type 436 enhanced luciferase activity via the MYH10 intronic region in MSR cells, the opposite effect was 437 observed in HEL cells. GATA1 is known to have both activating and repressing activity depending on 438 the cellular and promoter/enhancer context [40]. Variations in the expression of GATA1 partners in 439 these two cell models, such as RUNX1 and FLI1, may explain these differences. In MSR cells, the 440 GATA1 responsive activity decreased in presence of each variant, compared with wild-type GATA1, 441 indicating that the increase in MYH10 levels observed in patients likely results from reduced 442 interaction between the product of the GATA1 variants and the MYH10 intronic region. Intronic 443 GATA1 binding sites have already been shown to be involved in the regulation of 5-aminolevulinate 444 synthase 2 (ALAS2) gene expression, which is involved in heme synthesis in erythroid cells [41]. 445 In summary, we describe two novel pathogenic variations in the GATA1 gene characterized by distinct 446 clinical and laboratory findings. Our results suggest that variants affecting the GATA1 C-terminal ZF 
447 should likely be assessed in patients with normal platelet counts and platelet aggregation defects in 448 response to collagen and arachidonic. Importantly, Both GATA1 variants analyzed in this study led 449 to persistent expression of MYH10 in platelets. We identify a MYH10 intronic region that functions as 450 a GATA1-responsive cis-regulatory element. 


\section{Acknowledgments}

453 The study was funded by the "Fondation pour la Recherche Médicale" (granted to PS: 454 FDM20150633607), the French Foundation for Rare Diseases (grant WES 2012-2001), the National 455 Institutes of Diabetes, Digestive and Kidney diseases (NIDDK) of the National Institutes of Health 456 (granted to JVA: DK101628). The authors acknowledge the members of the French Reference Center 457 for Inherited Hereditary Platelet Disorders (CRPP) for their contribution to clinical analysis of the 458 patients and Dr. M Fiore for providing patient samples. 
460 Author contributions

461 PS, SA, and MaP designed and performed the experiments, analyzed the data and wrote the 462 manuscript. DP, DPB, MiP, FP and MC analyzed the data and revised the manuscript. NS performed 463 DNA sequencing and revised the manuscript. JCB performed TEM analysis and revised the 464 manuscript. JvA performed the structure analysis experiments and revised the manuscript. DB, ML, 465 CF and PEM performed clinical and biological characterization of patients, analyzed the data and 466 revised the manuscript. MP and MCA supervised the study and wrote the manuscript. 


\section{Declaration of interest}

469 The authors have declared that no conflict of interest exists.

470

471

472

473

474

475

476

477

478

479

480

481

482

483

484

485

486

487

488

489

490

491

492

493

494

495

496

497

498

499

\section{References}

1 Crispino JD. GATA1 in normal and malignant hematopoiesis. Semin Cell Dev Biol 2005; 16: 137-47.

2 Crispino JD, Horwitz MS. GATA factor mutations in hematologic disease. Blood 2017; 129: 2103-10.

3 Songdej N, Rao AK. Hematopoietic transcription factor mutations: important players in inherited platelet defects. Blood 2017; 129: 2873-81.

4 Ko LJ, Engel JD. DNA-binding specificities of the GATA transcription factor family. Mol Cell Biol 1993; 13: 4011-22.

5 Mehaffey MG, Newton AL, Gandhi MJ, Crossley M, Drachman JG. X-linked thrombocytopenia caused by a novel mutation of GATA-1. Blood 2001; 98: 2681-8.

6 Nichols KE, Crispino JD, Poncz M, White JG, Orkin SH, Maris JM, Weiss MJ. Familial dyserythropoietic anaemia and thrombocytopenia due to an inherited mutation in GATA1. Nat Genet 2000; 24: 266-70.

7 Chang AN, Cantor AB, Fujiwara Y, Lodish MB, Droho S, Crispino JD, Orkin SH. GATA-factor dependence of the multitype zinc-finger protein FOG-1 for its essential role in megakaryopoiesis. PNAS National Academy of Sciences; 2002; 99: 9237-42.

8 Shivdasani RA, Fujiwara Y, McDevitt MA, Orkin SH. A lineage-selective knockout establishes the critical role of transcription factor GATA-1 in megakaryocyte growth and platelet development. EMBO J 1997; 16: 3965-73.

9 Vyas P, Ault K, Jackson CW, Orkin SH, Shivdasani RA. Consequences of GATA-1 deficiency in megakaryocytes and platelets. Blood 1999; 93: 2867-75.

10 Muntean AG, Pang L, Poncz M, Dowdy SF, Blobel GA, Crispino JD. Cyclin D-Cdk4 is regulated by GATA-1 and required for megakaryocyte growth and polyploidization. Blood 2007; 109: 5199207.

11 Meinders M, Hoogenboezem M, Scheenstra MR, De Cuyper IM, Papadopoulos P, Németh T, Mócsai A, van den Berg TK, Kuijpers TW, Gutiérrez L. Repercussion of Megakaryocyte-Specific Gata1 Loss on Megakaryopoiesis and the Hematopoietic Precursor Compartment. PLoS ONE 2016; 11: e0154342. 
12 Mazzi S, Lordier L, Debili N, Raslova H, Vainchenker W. Megakaryocyte and polyploidization. Exp Hematol 2018; 57: 1-13.

502

503

504

505

506

507

508

509

510

511

512

513

514

515

516

517

518

519

520

521

522

523

524

525

526

527

528

529

530

531

532

533

534

535

13 Lordier L, Bluteau D, Jalil A, Legrand C, Pan J, Rameau P, Jouni D, Bluteau O, Mercher T, Leon C, Gachet C, Debili N, Vainchenker W, Raslova H, Chang Y. RUNX1-induced silencing of nonmuscle myosin heavy chain IIB contributes to megakaryocyte polyploidization. Nat Commun 2012; 3: 717.

14 Bluteau D, Glembotsky AC, Raimbault A, Balayn N, Gilles L, Rameau P, Nurden P, Alessi MC, Debili N, Vainchenker W, Heller PG, Favier R, Raslova H. Dysmegakaryopoiesis of FPD/AML pedigrees with constitutional RUNX1 mutations is linked to myosin II deregulated expression. Blood 2012; 120: 2708-18.

15 Antony-Debré I, Bluteau D, Itzykson R, Baccini V, Renneville A, Boehlen F, Morabito M, Droin $\mathrm{N}$, Deswarte C, Chang Y, Leverger G, Solary E, Vainchenker W, Favier R, Raslova H. MYH10 protein expression in platelets as a biomarker of RUNX1 and FLI1 alterations. Blood 2012; 120: 2719-22.

16 Stockley J, Morgan NV, Bem D, Lowe GC, Lordkipanidzé M, Dawood B, Simpson MA, Macfarlane K, Horner K, Leo VC, Talks K, Motwani J, Wilde JT, Collins PW, Makris M, Watson SP, Daly ME, UK Genotyping and Phenotyping of Platelets Study Group. Enrichment of FLI1 and RUNX1 mutations in families with excessive bleeding and platelet dense granule secretion defects. Blood 2013; 122: 4090-3.

17 Stevenson WS, Rabbolini DJ, Beutler L, Chen Q, Gabrielli S, Mackay JP, Brighton TA, Ward CM, Morel-Kopp M-C. Paris-Trousseau thrombocytopenia is phenocopied by the autosomal recessive inheritance of a DNA-binding domain mutation in FLI1. Blood 2015; 126: 2027-30.

18 Saultier P, Vidal L, Canault M, Bernot D, Falaise C, Pouymayou C, Bordet J-C, Saut N, Rostan A, Baccini V, Peiretti F, Favier M, Lucca P, Deleuze J-F, Olaso R, Boland A, Morange PE, Gachet C, Malergue F, Fauré $S$, et al. Macrothrombocytopenia and dense granule deficiency associated with FLI1 variants: ultrastructural and pathogenic features. Haematologica 2017; 102: 100616.

19 Tijssen MR, Cvejic A, Joshi A, Hannah RL, Ferreira R, Forrai A, Bellissimo DC, Oram SH, Smethurst PA, Wilson NK, Wang X, Ottersbach K, Stemple DL, Green AR, Ouwehand WH, Göttgens B. Genome-wide analysis of simultaneous GATA1/2, RUNX1, FLI1, and SCL binding in megakaryocytes identifies hematopoietic regulators. Dev Cell 2011; 20: 597-609.

20 Xu G, Kanezaki R, Toki T, Watanabe S, Takahashi Y, Terui K, Kitabayashi I, Ito E. Physical association of the patient-specific GATA1 mutants with RUNX1 in acute megakaryoblastic leukemia accompanying Down syndrome. Leukemia 2006; 20: 1002-8.

21 Elagib KE, Racke FK, Mogass M, Khetawat R, Delehanty LL, Goldfarb AN. RUNX1 and GATA-1 coexpression and cooperation in megakaryocytic differentiation. Blood 2003; 101: 4333-41. 
22 Kobayashi E, Shimizu R, Kikuchi Y, Takahashi S, Yamamoto M. Loss of the Gata1 gene IE exon leads to variant transcript expression and the production of a GATA1 protein lacking the Nterminal domain. J Biol Chem 2010; 285: 773-83.

23 Singleton BK, Roxby DJ, Stirling JW, Spring FA, Wilson C, Poole J, Anstee DJ. A novel GATA1 mutation (Stop414Arg) in a family with the rare X-linked blood group Lu(a-b-) phenotype and mild macrothrombocytic thrombocytopenia. Br J Haematol 2013; 161: 139-42.

24 Pereira J, Bento C, Manco L, Gonzalez A, Vagace J, Ribeiro ML. Congenital dyserythropoietic anemia associated to a GATA1 mutation aggravated by pyruvate kinase deficiency. Ann Hematol 2016; 95: 1551-3.

25 Yu C, Niakan KK, Matsushita M, Stamatoyannopoulos G, Orkin SH, Raskind WH. X-linked thrombocytopenia with thalassemia from a mutation in the amino finger of GATA-1 affecting DNA binding rather than FOG-1 interaction. Blood 2002; 100: 2040-5.

26 Balduini CL, Pecci A, Loffredo G, Izzo P, Noris P, Grosso M, Bergamaschi G, Rosti V, Magrini U, Ceresa IF, Conti V, Poggi V, Savoia A. Effects of the R216Q mutation of GATA-1 on erythropoiesis and megakaryocytopoiesis. Thromb Haemost 2004; 91: 129-40.

27 Hughan SC, Senis Y, Best D, Thomas A, Frampton J, Vyas P, Watson SP. Selective impairment of platelet activation to collagen in the absence of GATA1. Blood 2005; 105: 4369-76.

28 Åström M, Hahn-Strömberg V, Zetterberg E, Vedin I, Merup M, Palmblad J. X-linked thrombocytopenia with thalassemia displays bone marrow reticulin fibrosis and enhanced angiogenesis: comparisons with primary myelofibrosis. Am J Hematol 2015; 90: E44-48.

29 Robert P, Canault M, Farnarier C, Nurden A, Grosdidier C, Barlogis V, Bongrand P, Pierres A, Chambost $\mathrm{H}$, Alessi M-C. A novel leukocyte adhesion deficiency III variant: kindlin-3 deficiency results in integrin- and nonintegrin-related defects in different steps of leukocyte adhesion. $J$ Immunol 2011; 186: 5273-83.

30 Canault M, Ghalloussi D, Grosdidier C, Guinier M, Perret C, Chelghoum N, Germain M, Raslova $H$, Peiretti F, Morange PE, Saut N, Pillois X, Nurden AT, Cambien F, Pierres A, van den Berg TK, Kuijpers TW, Alessi M-C, Tregouet D-A. Human CalDAG-GEFI gene (RASGRP2) mutation affects platelet function and causes severe bleeding. J Exp Med 2014; 211: 1349-62.

31 White JG, Thomas A. Platelet structural pathology in a patient with the X-linked GATA-1, R216Q mutation. Platelets 2009; 20: 41-9.

32 Ferreira R, Ohneda K, Yamamoto M, Philipsen S. GATA1 function, a paradigm for transcription factors in hematopoiesis. Mol Cell Biol 2005; 25: 1215-27.

33 Liu Z-J, Italiano J Jr, Ferrer-Marin F, Gutti R, Bailey M, Poterjoy B, Rimsza L, Sola-Visner M. Developmental differences in megakaryocytopoiesis are associated with up-regulated TPO signaling through mTOR and elevated GATA-1 levels in neonatal megakaryocytes. Blood 2011; 117: 4106-17. 
34 Cai W, Huang J, Zhu Q, Li BE, Seruggia D, Zhou P, Nguyen M, Fujiwara Y, Xie H, Yang Z, Hong D, Ren P, Xu J, Pu WT, Yuan G-C, Orkin SH. Enhancer dependence of cell-type-specific gene expression increases with developmental age. Proc Natl Acad Sci U S A 2020; 117: 21450-8.

575

576

577

578

579

580

581

582

583

584

585

586

587

588

589

590

591

592

593

594

595

596

597

598

599

600

601

602

603

604

605

606

607

608

609

610

35 Poggi M, Canault M, Favier M, Turro E, Saultier P, Ghalloussi D, Baccini V, Vidal L, Mezzapesa A, Chelghoum N, Mohand-Oumoussa B, Falaise C, Favier R, Ouwehand WH, Fiore M, Peiretti F, Morange PE, Saut N, Bernot D, Greinacher A, et al. Germline variants in ETV6 underlie reduced platelet formation, platelet dysfunction and increased levels of circulating CD34+ progenitors. Haematologica 2017; 102: 282-94.

36 Shin E, Jeong J-G, Chung H, Jung H, Park C, Yoon SR, Kim T-D, Lee SJ, Choi I, Noh J-Y. The Gata1low murine megakaryocyte-erythroid progenitor cells expand robustly and alter differentiation potential. Biochem Biophys Res Commun 2020; 528: 46-53.

37 Yang F, Wei Q, Adelstein RS, Wang PJ. Non-muscle myosin IIB is essential for cytokinesis during male meiotic cell divisions. Dev Biol 2012; 369: 356-61.

38 Badirou I, Pan J, Legrand C, Wang A, Lordier L, Boukour S, Roy A, Vainchenker W, Chang Y. Carboxyl-terminal-dependent recruitment of nonmuscle myosin II to megakaryocyte contractile ring during polyploidization. Blood 2014; 124: 2564-8.

39 Eisbacher M, Holmes ML, Newton A, Hogg PJ, Khachigian LM, Crossley M, Chong BH. Proteinprotein interaction between Fli-1 and GATA-1 mediates synergistic expression of megakaryocyte-specific genes through cooperative DNA binding. Mol Cell Biol 2003; 23: 342741.

40 Welch JJ, Watts JA, Vakoc CR, Yao Y, Wang H, Hardison RC, Blobel GA, Chodosh LA, Weiss MJ. Global regulation of erythroid gene expression by transcription factor GATA-1. Blood 2004; 104: 3136-47.

41 Zhang Y, Zhang J, An W, Wan Y, Ma S, Yin J, Li X, Gao J, Yuan W, Guo Y, Engel JD, Shi L, Cheng T, Zhu X. Intron 1 GATA site enhances ALAS2 expression indispensably during erythroid differentiation. Nucleic Acids Res 2017; 45: 657-71.

\section{Figure legend}

\section{Figure 1. Identification of two novel GATA1 variants.}

A) Pedigrees of the affected families. Squares denote males, and circles represent females. The slashes denote deceased family members. Solid black symbols represent affected family members carrying a hemizygous GATA1 variant. Circles with a dot represent heterozygous female carriers of the variants. Solid gray symbols represent non-tested family members with a medical history of bleeding events.

(B) Sanger sequencing electropherograms of the indicated family members.

(C) Schematic diagram of the GATA1 protein domain structure. The functional N-terminal transactivation domain (TD) and both the $\mathrm{N}$ - and $\mathrm{C}$-terminal zinc-finger domains (ZF1 and ZF2, respectively) are depicted. The positions of the variations within GATA1 are indicated in red (variations reported in this study) and black (variations reported in previous studies). 
(D) Left: Figure. Structure of Zn fingers of GATA1 bound to palindromic DNA recognition site (PDB

612

613

614

615

616

617

618

619

620

621

622

623

624

625

626

627

628

629

630

631

632

633

634

635

636

637

638

639

640

641

642

643

644

645

646

647

648

649

650

651

652

653

654

655

656

657

658

659

660

661

662

663 code: 3VD6). The diagram shows GATA1 in green cartoon with key residues L268 and N206 in yellow sticks, and the DNA fragment in magenta sticks. Zn2+ ions are in grey spheres with stick representation of coordinating residues. Other GATA1 relevant variants V205M and G208R are mapped in orange sticks. Inset A show 3VD6 superimposed to the same structure harboring a model of the L268M mutation. Right: Lowest energy conformer of NMR structure of Zn fingers of GATA1 bound to FOG1 (PDB code: 1Y0J). The diagram shows GATA1 in green cartoon with key residue N206 in yellow stick, and FOG1 in red. Zn2+ ions are in grey spheres with stick representation of coordinating residues. Other GATA1 relevant variants V205M and G208R are mapped in orange sticks. Inset A shows 1Y0J superimposed to the same structure harboring a model of the N206I mutation. In dashes is shown an atomic clash between I206 (GATA1) and Y30 (FOG1) with interatomic distances below $2.5 \AA$.

Figure 2. Quantitative and morphological modifications of platelets and red blood cells in GATA1 variant carriers

(A) Evolution of platelet count and mean corpuscular volume (MCV) of erythrocytes with age in the two F2 probands.

(B) Representative images of peripheral blood smears stained with May-Grünwald-Giemsa for patients F1-II-1 and F2-II-2 (at 6 and 23 years old, respectively). The peripheral blood smear images revealed hypogranular platelets (left, black arrow, magnification $\times 1000$ ) mild anisocytosis and poikylocytosis (right, magnification x 500).

(C) Representative images of bone marrow aspirate smears stained with May-Grünwald-Giemsa for patient F1-II-1 (at 17 months old). Assessment of bone marrow aspirates revealed dysmegakaryopoiesis with hypolobulated megakaryocytes (left, black arrow, magnification $\times 200$ ) and dyserythropoiesis with nuclear karyorrhexis (right, black arrow, magnification x500). Smears were examined for each subject by a well-trained hematologist (M Loosveld).

\section{Figure 3. Alpha and dense granule defects associated with GATA1 variants.}

(A) Representative micrographs of dense granule distribution in a control and patients F1-II-1 (at 8 years old), F2-II-1 (at 27 years old) and F2-II-2 (at 23 years old). Scale bar: $1 \mu \mathrm{m}$. The graph shows the cumulative distribution of dense granules per whole platelet deposited on formar film for patient vs. control platelets (healthy subjects, $n=54$ ); 100 platelets per whole-platelet mount were examined for each subject by a well-trained engineer blinded to the genotype (JC Bordet, Unité d'Hémostase Biologique, Bron, France).

(B) Platelet sections from a healthy subject (control panel) and patient F2-II-1 (all other panels). Platelet section from control shows numerous alpha granules, mitochondria recognizable by the crests, and a large glycogen area. Although the numbers are variable, platelets derived from patient F2-II-1 at 22 years old displayed low alpha granule counts for each section, sometimes lower than mitochondria counts. Overall, the sections display either very few organelles with a relatively amorphous background (i) or normal counts for organelles that show variable granulation (iv). Other notable structures observed included very large areas of membrane complexes (ii, iii) and apoptotic granules (left part of panel iii). We also observed membranous formations that resemble a dense tubular system, although the formations were occasionally arranged in a large ring (i), in the vicinity of the tubulin circle (bottom center panel) or in aggregates in the cytoplasm (v). Scale bar: $0.5 \mu \mathrm{m}$. The graphs show the cumulative distribution of platelet area, number of alpha granules $/ \mu \mathrm{m}^{2}$, major platelet diameter, and alpha granule diameter using classical electron microscopy sections in patient F2-II-1 vs. control platelets (healthy subjects $n=10$ ); we examined 100 platelets per section.

Figure 4. Increased number of $\mathrm{CD}_{34}{ }^{+}$cells and abnormal megakaryocyte differentiation in GATA1 variant carriers.

(A) Flow cytometry analysis of circulating CD34+ cells. Left: Representative dot plots of cells from one healthy control and patient F1-II-1. The plots display the side scatter and CD34 expression analysis in CD19low CD45low cells. The ellipse gates show the CD19low CD45low CD34+ cell population. Right: The percentage of CD34+ cells in the total CD19low CD45low cell population. The control data 
represent five different individuals. The data for patient F1-II-1 was obtained from two independent experiments carried out at 15 -month intervals. The data are provided as the median \pm interquartile range.

667

668

(B-D) In vitro megakaryocyte differentiation. Circulating $\mathrm{CD}^{4} 4^{+}$cells from F1-II-1 patient or controls were isolated and cultured in the presence of TPO and SCF to induce megakaryocytic commitment. (B) Megakaryocyte differentiation was monitored via flow cytometry. The density plots display the CD41/CD42a expression profiles of two unrelated controls and F1-II-1 affected patient (at days 9 and 11 of differentiation). (C) Representative images of cultured cells at day 11 (20X magnification). The graph represents the median \pm interquartile range of the cell surface area. Fifty cells were quantified. Differences between the groups were assessed using the Kruskal-Wallis test followed by Dunn's multiple comparison test. (D) Ploidy levels were monitored via flow cytometry. The histograms display the frequency distribution of Hoechst 33342 levels in CD34+-derived cells from two unrelated controls and F1-II-1 affected patient at day 9 of differentiation.

(E) Western blot analysis of MYH10 expression in platelets from the patients with GATA1 variants (F1-II2, F2-II-1 and F2-II-2), one heterozygous carrier (F2-I-1), one unaffected family member (F2-I2) and four unrelated controls. Platelets from a patient carrying the FLI1 p R337Q variant represents the MYH10 positive control. GAPDH was used as a protein loading control. Quantification of band intensity for MYH10 is shown below the western blot.

Figure 5. Identification and functional analysis of the MYH10 regulatory regions.

684

685

686

687

688

689

690

691

692

693

694

695

696

697

698

699

700

701

702

703

704

705

706

707

708

709

710

(A) Visualization of $M Y H 10$ regulatory regions with GATA1, FLI1 and RUNX1 binding peaks identified via ChIP-sequencing. Part of the NDEL gene is shown upstream of the MYH10 gene using the UCSC genome browser (chr17:8,435,074-8,669,900 using GRCh38/hg38). The bottom layer shows the position of two GATA1 binding region peaks within the 3'UTR (untranslated transcribed region, blue arrows) and intron 8 (red arrows) of MYH10 for the transcription factors GATA1, FLI1 and RUNX1.

(B) Luciferase expression in HEL cells transfected with different pGL3 luciferase reporter vectors including the 3'UTR or intron 8 regions of $M Y H 10$ gene. Mutations on GATA1-binding sites in intron 8 were introduced as indicated (M1 or M2 or M3 or $M(1,2,3,4)$ ). The pGL3 vector without regulatory regions (empty vector) was used as a control. Each plasmid was assayed in three to seven independent transfection experiments. The dual luciferase assay was performed by sequentially measuring the firefly and renilla luciferase activities of the same sample with the results expressed as the ratio of firefly to renilla activity (Fluc/Rluc). ${ }^{* *}$ : $p<0.001,{ }^{* * *}: p<0.0001 \mathrm{vs.} \mathrm{pGL3-intron} 8$ MYH10 wild-type (WT) (one-way ANOVA corrected for multiple comparisons). +++: $p<0.0001$ vs. pGL3-empty vector (one-way ANOVA corrected for multiple comparisons).

(C) Luciferase expression in GripTite 293 MSR cells transfected with different pGL3 luciferase reporters and pCDNA3-GATA1 vectors as indicated. Left: pGL3-intron 8 MYH10 wild-type (WT), pGL3-intron 8 MYH10 mutants, empty pCDNA3 and pCDNA3-GATA1 WT. Middle: pGL3-3'UTR MYH10, empty pCDNA3 and pCDNA3-GATA1 WT. Right: empty pGL3, empty pCDNA and pCDNAGATA1 WT. The pCDNA3 empty vector was used as a control. *: $p<0.01$, ${ }^{* * * *}: p<0.0001$ vs. pGL3intron8-MYH10 WT in the presence of GATA1 (black bar) (one-way ANOVA corrected for multiple comparisons). ++++: $p<0.0001$ vs. the empty pGL3 vector in the absence of GATA1 (white bar). Each plasmid was assayed in six separate transfection experiments (one-way ANOVA corrected for multiple comparisons).

(D) Luciferase expression after transfection in GripTite 293 MSR cells with pGL3-intron 8 MYH10 WT and pCDNA3-GATA1 WT or variant vectors as indicated. *: $p<0.01$ vs. GATA1 WT (one-way ANOVA corrected for multiple comparisons). Each plasmid was assayed in five separate transfection experiments.

711 


\section{Table 1. Platelet aggregation assays}

713 Results correspond to assays performed in 1990 and 1993 (A) and 2015 (B).

714
A 715

\begin{tabular}{|c|c|c|c|c|c|c|c|c|c|}
\hline \multirow{3}{*}{ Individuals } & \multirow{3}{*}{$\begin{array}{c}\text { Age } \\
\text { (years) }\end{array}$} & \multirow{3}{*}{$\begin{array}{c}\text { Blood } \\
\text { platelet } \\
\text { count } \\
\left(\times 10^{9} / l\right)\end{array}$} & \multirow{3}{*}{$\begin{array}{c}\text { PRP platelet } \\
\text { count } \\
\left(\times 10^{9} / 1\right)\end{array}$} & \multicolumn{6}{|c|}{ Platelet aggregation maximal intensity (\%) } \\
\hline & & & & \multicolumn{2}{|c|}{ ADP } & \multicolumn{2}{|c|}{ Collagen } & \multirow{2}{*}{$\begin{array}{c}\text { Arachidonic acid } \\
\qquad 0.5 \mathrm{mg} / \mathrm{ml}\end{array}$} & \multirow{2}{*}{$\begin{array}{r}\text { Ristocetine } \\
1.5 \mathrm{mg} / \mathrm{ml}\end{array}$} \\
\hline & & & & $1 \mu \mathrm{M}$ & $2.5 \mu \mathrm{M}$ & $0.36 \mu \mathrm{g} / \mathrm{ml}$ & $0.72 \mu \mathrm{g} / \mathrm{ml}$ & & \\
\hline Control * & NA & NA & 350 & 45 & 68 & 60 & 63 & 69 & 76 \\
\hline F2-II-1 & 1.7 & 233 & 380 & 9 & 25 & 0 & 0 & 19 & 67 \\
\hline Control * & NA & NA & 350 & 45 & 68 & 57 & 63 & 69 & 68 \\
\hline F2-II-2 & 0.8 & 225 & 338 & 8 & 23 & 0 & 0 & 11 & 46 \\
\hline
\end{tabular}

716

717

718

B 719

\begin{tabular}{|c|c|c|c|c|c|c|c|c|c|}
\hline \multirow{3}{*}{ Individuals } & \multirow{3}{*}{$\begin{array}{c}\text { Age } \\
\text { (years) }\end{array}$} & \multirow{3}{*}{$\begin{array}{l}\text { Blood } \\
\text { platelet } \\
\text { count } \\
\text { (x 109/I) }\end{array}$} & \multirow{3}{*}{$\begin{array}{c}\text { PRP } \\
\text { platelet } \\
\text { count } \\
\text { (x 109/I) }\end{array}$} & \multicolumn{6}{|c|}{ Platelet aggregation maximal intensity (\%) } \\
\hline & & & & & ADP & & Collagen & Arachidonic acid & Ristocetine \\
\hline & & & & $2.5 \mu \mathrm{M}$ & $5 \mu \mathrm{M}$ & $10 \mu \mathrm{M}$ & $3.3 \mu \mathrm{g} / \mathrm{ml}$ & $0.5 \mathrm{mg} / \mathrm{ml}$ & $1.25 \mathrm{mg} / \mathrm{ml}$ \\
\hline $\begin{array}{c}\text { Normal } \\
\text { range }\end{array}$ & & & & $83-93$ & $75-90$ & $78-92$ & $79-85$ & $76-91$ & $78-948$ \\
\hline F2II-1 & 26.7 & 106 & 159 & 40 & 62 & 56 & 25 & 14 & 73 \\
\hline F2II-2 & 22.7 & 58 & 102 & 40 & 62 & 68 & 24 & 11 & 70 \\
\hline
\end{tabular}

720

721 * Controls correspond to one healthy individual tested at the same time.

722 
1 Table 2. Platelet glycoprotein expression

2

\begin{tabular}{|c|c|c|c|c|c|c|}
\hline Platelet glycoprotein MFI (a.u.) & & F1-II-1 & $\begin{array}{c}\text { Normal range } \\
\left(10^{\text {th }}-90^{\text {th }} \text { range } n=14\right)\end{array}$ & $\begin{array}{l}\text { ndividual } \\
\text { F2-II-1 }\end{array}$ & F2-II-2 & $\begin{array}{c}\text { Normal range } \\
\left(10^{\text {th-90th }} \text { range } n=12\right)\end{array}$ \\
\hline \multirow{2}{*}{ allbß3 (CD41) } & Basal & 24 & $21-44$ & 24 & 30 & $20-29$ \\
\hline & TRAP14 50 $\mu \mathrm{M}$ & 25 & $30-62$ & 30 & 34 & $28-46$ \\
\hline \multirow{2}{*}{ GPIba (CD42b) } & Basal & 1.7 & $1.2-.3$ & 2.1 & 2.4 & $1.2-2.4$ \\
\hline & TRAP14 50 $\mu \mathrm{M}$ & 1.5 & $0.9-2.3$ & 1.8 & 1.8 & $0.6-1.1$ \\
\hline \multirow{3}{*}{ CD63 } & Basal & 1.2 & $0.9-3.4$ & 1.1 & 1.3 & $0.5-1$ \\
\hline & TRAP14 50 $\mu \mathrm{M}$ & 1.6 & $1.9-6.3$ & 1.7 & 1.3 & $1.3-3.6$ \\
\hline & Ratio TRAP-14 / basal & 1.3 & $1.7-3.7$ & 1.4 & 1 & $1.5-4.8$ \\
\hline \multirow{3}{*}{ CD62P (P-selectin) } & Basal & 0.2 & $0.09-0.35$ & 0.2 & 0.4 & $0.15-0.75$ \\
\hline & TRAP14 50 $\mu \mathrm{M}$ & 0.2 & $1.9-6.3$ & 1.5 & 1.7 & 2.3-5 \\
\hline & Ratio TRAP-14 / basal & 1.1 & $12.4-22.8$ & 7 & 4.2 & $5.5-25$ \\
\hline
\end{tabular}


1 Table 3. Characteristics of GATA1 variant carriers

\begin{tabular}{|c|c|c|}
\hline & Family 1 (F1-II-1) & Family 2 (F2-II-1 and F2-II-2) \\
\hline DNA change & c. $802 C>A$ & $\mathrm{C} 617 \mathrm{~A}>\mathrm{T}$ \\
\hline Protein change & p.N206I & p.L268M \\
\hline Variant localization & $\mathrm{N}$-terminal zinc finger & C-terminal zinc finger \\
\hline
\end{tabular}

Clinical

manifestations

Thrombocytopenia

Early in life (18 months) with a severely reduced platelet count (15 G/L).

Bleeding Frequent bruising, post vaccinal hematoma.

Bleeding during dental avulsion prevented by platelet concentrates.

Platelet Not done

Initially absent (at 18 and 9 months, 261 and 233 $\mathrm{G} / \mathrm{L}$ for each brother). Thrombocytopenia gradually developed from 2-5 years of age and then steadily worsened.

Pronounced mucocutaneous bleedings.

Bleeding during dental avulsion and appendicectomy was prevented by platelet concentrates.

Bleeding time $>15 \mathrm{~min}$

No aggregation test performed (because platelet number is $<20 \mathrm{G} / \mathrm{L}$ ).

Aggregation defect (mainly collagen and arachidonic acid) in absence of thrombocytopenia.

$>90 \%$ reduction in dense granule number.

Surface glycoproteins: No basal defect in CD41, $\mathrm{CD} 42 \mathrm{~b}, \mathrm{CD} 62 \mathrm{P}$, and $\mathrm{CD} 63$; reduced expression levels of CD63 and CD62P after TRAP-14 stimulation.

Serotonin: NA (too low platelet count).

PAl-1 antigen: normal value, $0.35 \mathrm{ng} / 10^{6}$ platelets (Normal values: 0.33-1.07).

Coagulation and fibrinolysis No defect.

Erythrocyte

Absence of anemia.

MCV : 77-81 fL (Normal values : 80-95).

Discrete anisopoïkilocytosis, few dacryocytes and schizocytes.

Fetal hemoglobin not evaluated.

Bone marrow Dysmegakaryopoiesis with hypolobulated megakaryocytes and mild dyserythropoiesis with nuclear karyorrhexis. 
1

2

3

4

5

6

7

8

9

10

11

12

13

14

15

16

17

18

19

20

21

22

24

25

26

27

28

29

30

31

32

33

34

35

36

37

38

39

40

41

42

43

44

45

46

47

48

49

50

51

52

53

54

55

56

57

58

59

60 
A

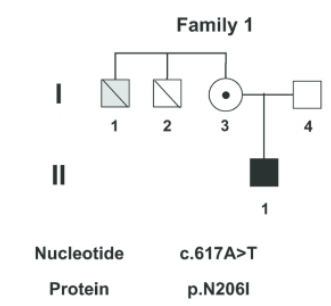

\section{FIGURE 1}

c

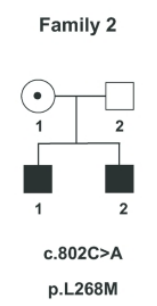

B
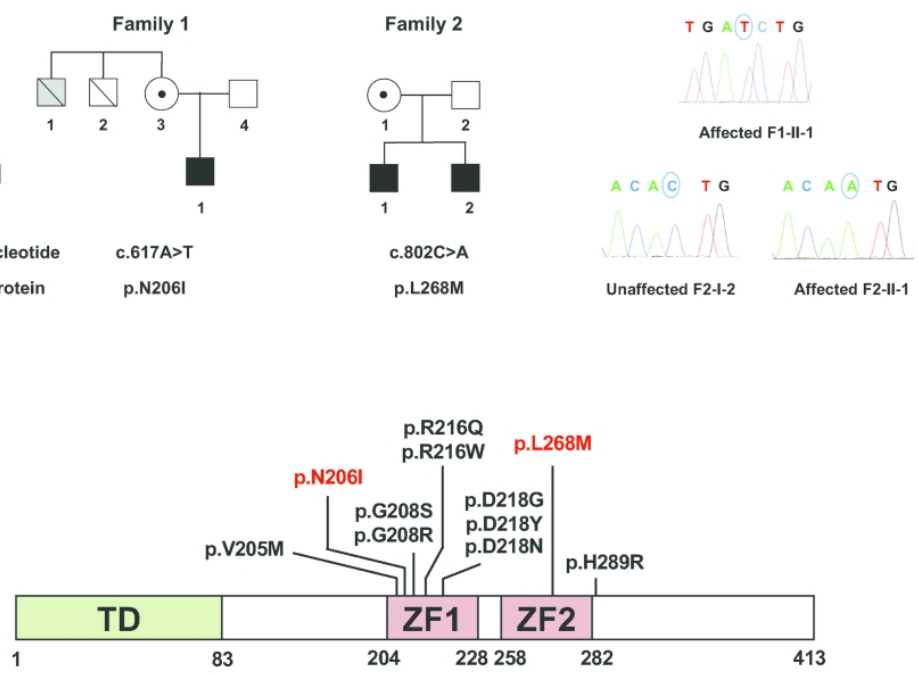

D
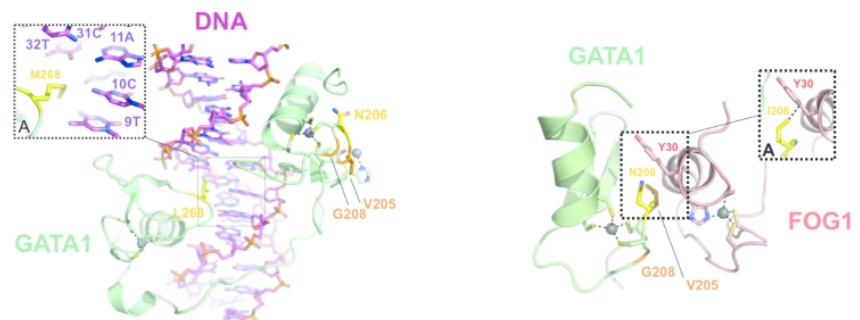

Figure 1. Identification of two novel GATA1 variants.

A) Pedigrees of the affected families. Squares denote males, and circles represent females. The slashes denote deceased family members. Solid black symbols represent affected family members carrying a hemizygous GATA1 variant. Circles with a dot represent heterozygous female carriers of the variants. Solid gray symbols represent non-tested family members with a medical history of bleeding events.

(B) Sanger sequencing electropherograms of the indicated family members.

(C) Schematic diagram of the GATA1 protein domain structure. The functional N-terminal transactivation domain (TD) and both the N- and C-terminal zinc-finger domains (ZF1 and ZF2, respectively) are depicted. The positions of the variations within GATA1 are indicated in red (variations reported in this study) and black (variations reported in previous studies). 
2

3

4

5

6

7

8

9

10

11

12

13

14

15

16

17

18

19

20

21

22

23

24

25

26

27

28

29

30

31

32

33

34

35

36

37

38

39

40

41

42

43

44

45

46

47

48

49

50

51

52

53

54

55

56

57

58

59

60 
A

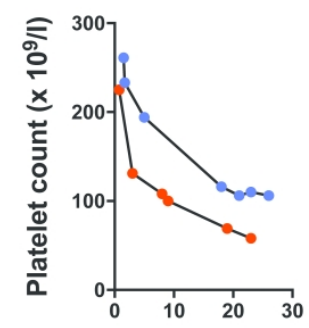

FIGURE 2

B
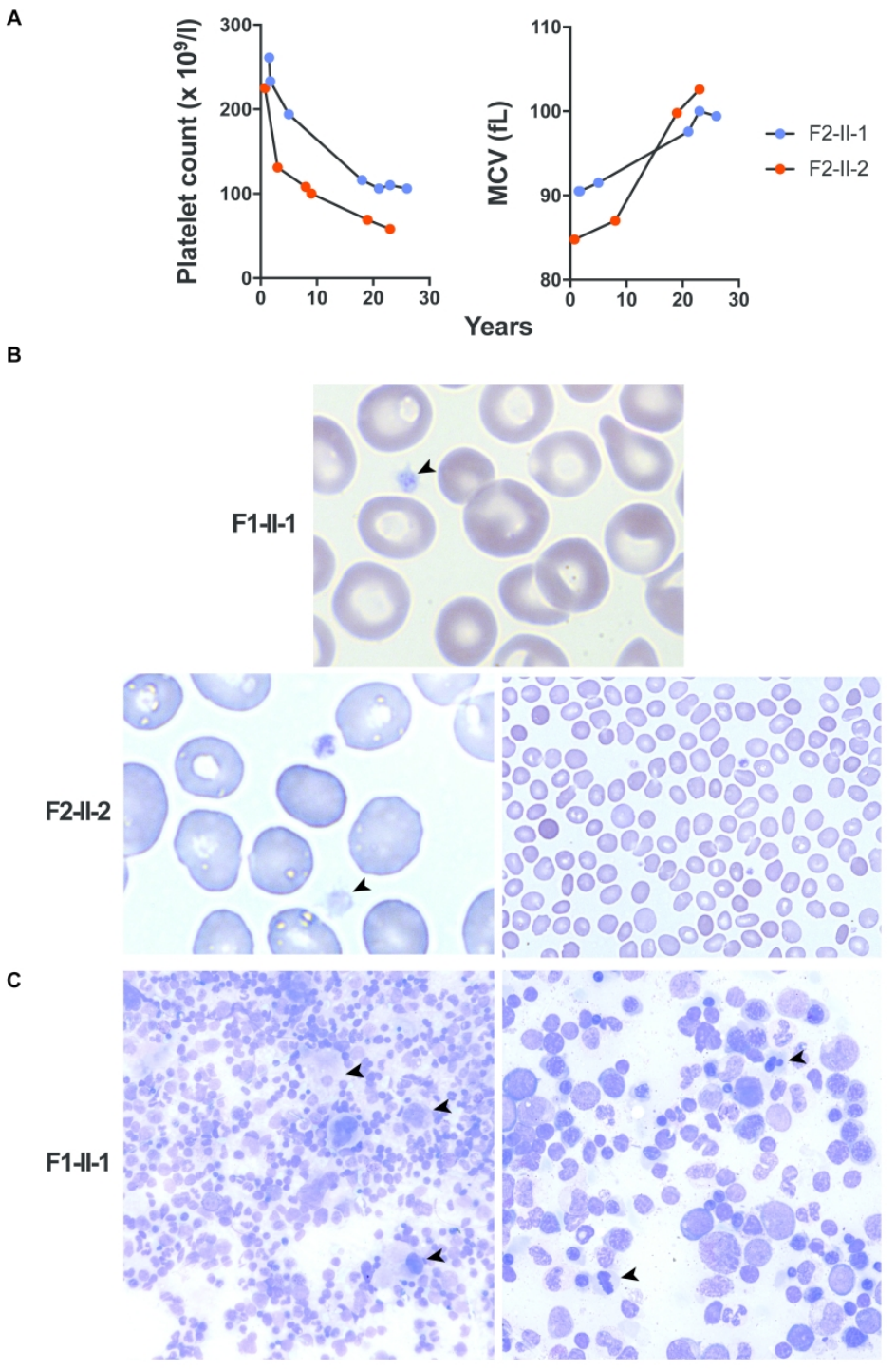

Figure 2. Quantitative and morphological modifications of platelets and red blood cells in GATA1 variant carriers

(A) Evolution of platelet count and mean corpuscular volume (MCV) of erythrocytes with age in the two F2 probands.(B) Representative images of peripheral blood smears stained with May-Grünwald-Giemsa for patients F1-II-1 and F2-II-2 (at 6 and 23 years old, respectively). The peripheral blood smear images revealed hypogranular platelets (left, black arrow, magnification $\times 1000$ ) mild anisocytosis and poikylocytosis (right, magnification x 500).(C) Representative images of bone marrow aspirate smears stained with MayGrünwald-Giemsa for patient F1-II-1 (at 17 months old). Assessment of bone marrow aspirates revealed dysmegakaryopoiesis with hypolobulated megakaryocytes (left, black arrow, magnification x 200) and dyserythropoiesis with nuclear karyorrhexis (right, black arrow, magnification x500). Smears were examined for each subject by a well-trained hematologist (M Loosveld). 


\section{FIGURE 3}

A

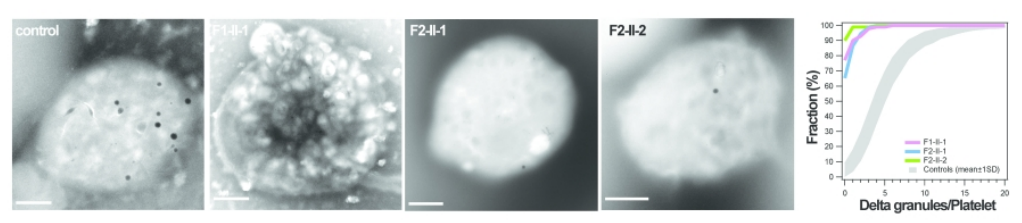

B
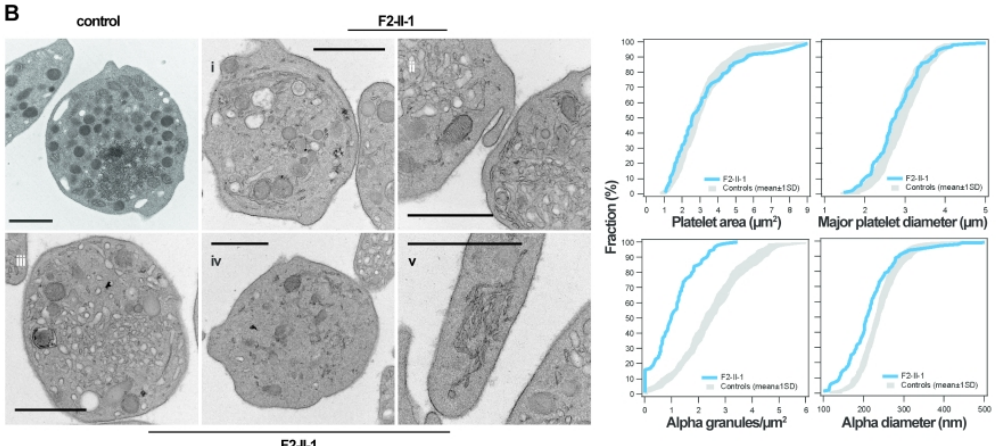

!" 岟\$!

Figure 3. Alpha and dense granule defects associated with GATA1 variants.

(A) Representative micrographs of dense granule distribution in a control and patients F1-II-1 (at 8 years old), F2-II-1 (at 27 years old) and F2-II-2 (at 23 years old). Scale bar: $1 \mu \mathrm{m}$. The graph shows the cumulative distribution of dense granules per whole platelet deposited on formar film for patient vs. control platelets (healthy subjects, $n=54$ ); 100 platelets per whole-platelet mount were examined for each subject by a well-trained engineer blinded to the genotype (JC Bordet, Unité d'Hémostase Biologique, Bron, France). (B) Platelet sections from a healthy subject (control panel) and patient F2-II-1 (all other panels). Platelet section from control shows numerous alpha granules, mitochondria recognizable by the crests, and a large glycogen area. Although the numbers are variable, platelets derived from patient F2-II-1 at 22 years old displayed low alpha granule counts for each section, sometimes lower than mitochondria counts. Overall, the sections display either very few organelles with a relatively amorphous background (i) or normal counts for organelles that show variable granulation (iv). Other notable structures observed included very large areas of membrane complexes (ii, iii) and apoptotic granules (left part of panel iii). We also observed membranous formations that resemble a dense tubular system, although the formations were occasionally arranged in a 
large ring (i), in the vicinity of the tubulin circle (bottom center panel) or in aggregates in the cytoplasm (v). Scale bar: $0.5 \mu \mathrm{m}$. The graphs show the cumulative distribution of platelet area, number of alpha granules/ $\mu \mathrm{m} 2$, major platelet diameter, and alpha granule diameter using classical electron microscopy sections in patient F2-II-1 vs. control platelets (healthy subjects $n=10$ ); we examined 100 platelets per section. 


\section{FIGURE 4}

A

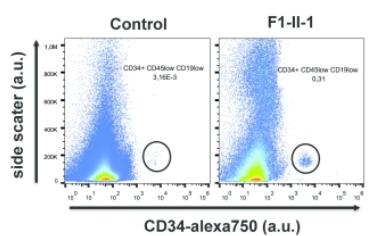

B

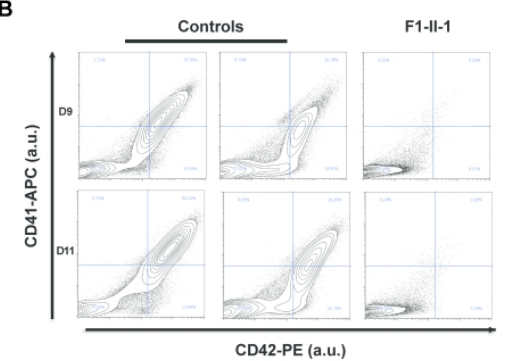

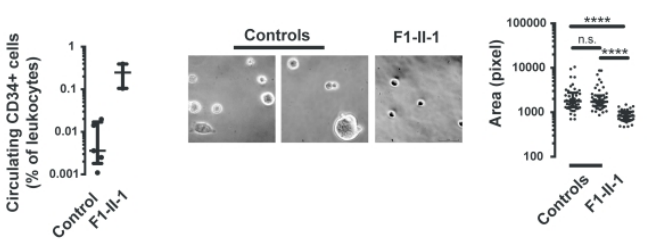

D

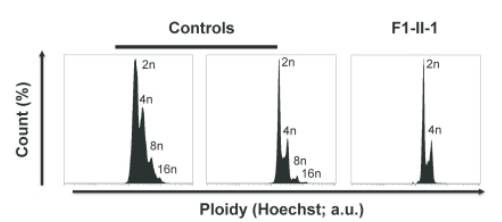

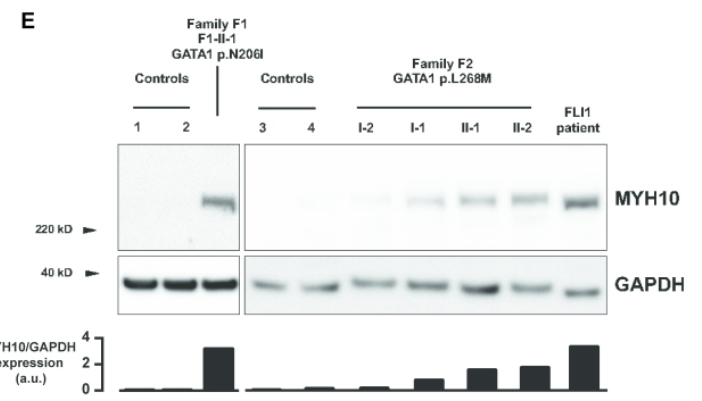

Figure 4. Increased number of CD34+ cells and abnormal megakaryocyte differentiation in GATA1 variant carriers.

(A) Flow cytometry analysis of circulating CD34+ cells. Left: Representative dot plots of cells from one healthy control and patient F1-II-1. The plots display the side scatter and CD34 expression analysis in CD19low CD45low cells. The ellipse gates show the CD19low CD45low CD34+ cell population. Right: The percentage of CD34+ cells in the total CD19low CD45low cell population. The control data represent five different individuals. The data for patient F1-II-1 was obtained from two independent experiments carried out at 15 -month intervals. The data are provided as the median \pm interquartile range.

(B-D) In vitro megakaryocyte differentiation. Circulating CD34+ cells from F1-II-1 patient or controls were isolated and cultured in the presence of TPO and SCF to induce megakaryocytic commitment. (B) Megakaryocyte differentiation was monitored via flow cytometry. The density plots display the CD41/CD42a expression profiles of two unrelated controls and F1-II-1 affected patient (at days 9 and 11 of differentiation). (C) Representative images of cultured cells at day 11 (20X magnification). The graph represents the median \pm interquartile range of the cell surface area. Fifty cells were quantified. Differences 
between the groups were assessed using the Kruskal-Wallis test followed by Dunn's multiple comparison test. (D) Ploidy levels were monitored via flow cytometry. The histograms display the frequency distribution of Hoechst 33342 levels in CD34+-derived cells from two unrelated controls and F1-II-1 affected patient at day 9 of differentiation.

(E) Western blot analysis of MYH10 expression in platelets from the patients with GATA1 variants (F1-II2, F2-II-1 and F2-II-2), one heterozygous carrier (F2-I-1), one unaffected family member (F2-I-2) and four unrelated controls. Platelets from a patient carrying the FLI1 p R337Q variant represents the MYH10 positive control. GAPDH was used as a protein loading control. Quantification of band intensity for MYH10 is shown below the western blot. 
FIGURE 5

A

B

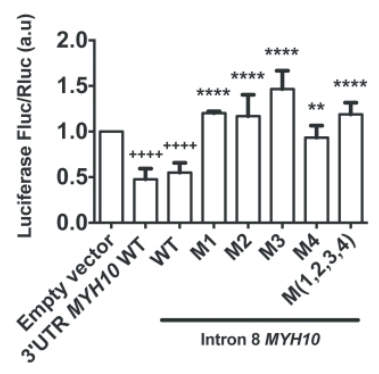

C
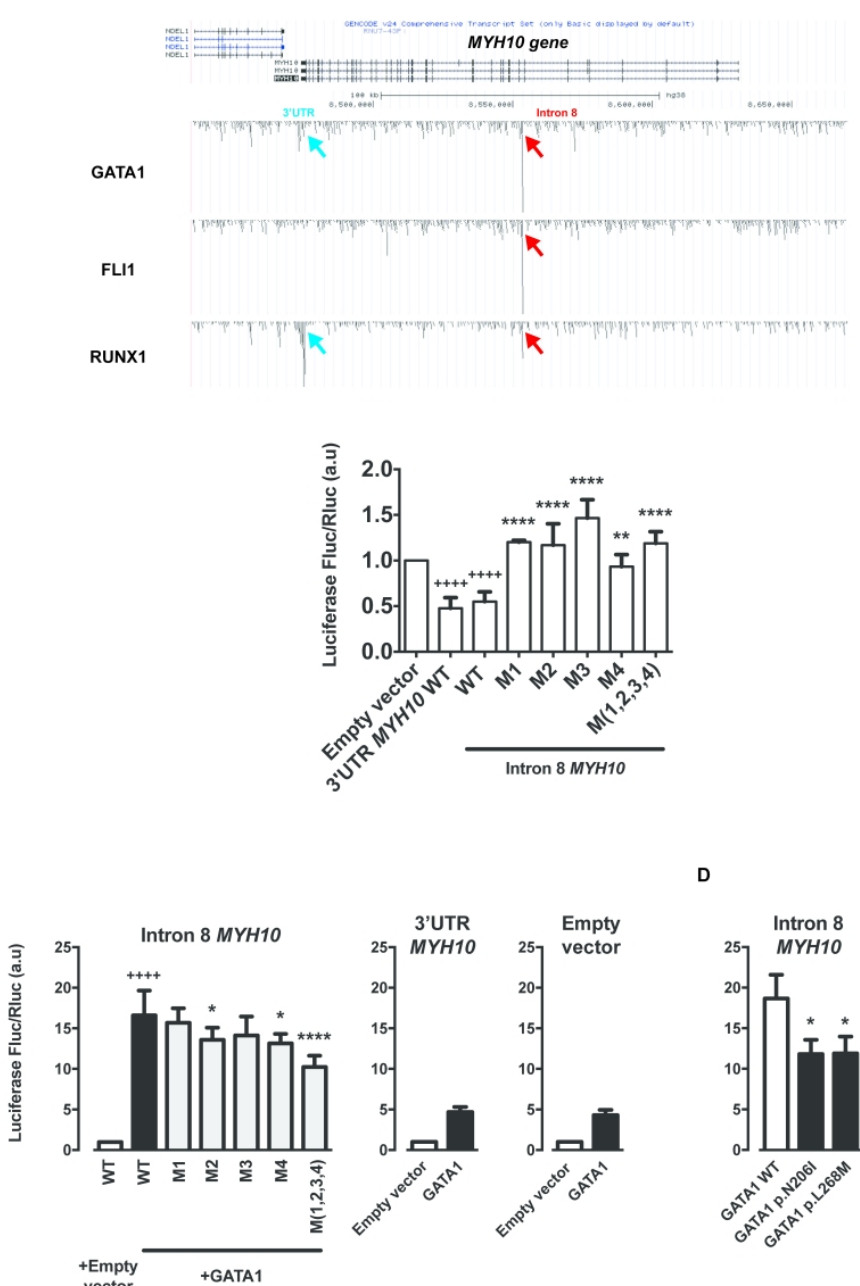

Figure 5. Identification and functional analysis of the MYH10 regulatory regions.

(A) Visualization of MYH10 regulatory regions with GATA1, FLI1 and RUNX1 binding peaks identified via ChIP-sequencing. Part of the NDEL gene is shown upstream of the MYH10 gene using the UCSC genome browser (chr17:8,435,074-8,669,900 using GRCh38/hg38). The bottom layer shows the position of two GATA1 binding region peaks within the $3^{\prime} \mathrm{UTR}$ (untranslated transcribed region, blue arrows) and intron 8 (red arrows) of MYH10 for the transcription factors GATA1, FLI1 and RUNX1.

(B) Luciferase expression in HEL cells transfected with different PGL3 luciferase reporter vectors including the $3^{\prime} U T R$ or intron 8 regions of MYH10 gene. Mutations on GATA1-binding sites in intron 8 were introduced as indicated ( $\mathrm{M} 1$ or $\mathrm{M} 2$ or $\mathrm{M} 3$ or $\mathrm{M}(1,2,3,4)$ ). The $\mathrm{pGL3}$ vector without regulatory regions (empty vector) was used as a control. Each plasmid was assayed in three to seven independent transfection experiments. The dual luciferase assay was performed by sequentially measuring the firefly and renilla luciferase activities of the same sample with the results expressed as the ratio of firefly to renilla activity (Fluc/Rluc). **: $\mathrm{p}<0.001, * * *: \mathrm{p}<0.0001$ vs. pGL3-intron $8 \mathrm{MYH10}$ wild-type (WT) (one-way ANOVA corrected for multiple comparisons). $+++:$ p $<0.0001$ vs. pGL3-empty vector (one-way ANOVA corrected for multiple 
comparisons).

(C) Luciferase expression in GripTite 293 MSR cells transfected with different pGL3 luciferase reporters and pCDNA3-GATA1 vectors as indicated. Left: pGL3-intron 8 MYH10 wild-type (WT), pGL3-intron 8 MYH10 mutants, empty pCDNA3 and pCDNA3-GATA1 WT. Middle: pGL3-3'UTR MYH10, empty pCDNA3 and pCDNA3-GATA1 WT. Right: empty pGL3, empty pCDNA and pCDNA-GATA1 WT. The pCDNA3 empty vector was used as a control. *: p<0.01, ****: p<0.0001 vs. pGL3-intron8-MYH10 WT in the presence of GATA1 (black bar) (one-way ANOVA corrected for multiple comparisons). $++++: p<0.0001$ vs. the empty pGL3 vector in the absence of GATA1 (white bar). Each plasmid was assayed in six separate transfection experiments (one-way ANOVA corrected for multiple comparisons).

(D) Luciferase expression after transfection in GripTite 293 MSR cells with pGL3-intron 8 MYH10 WT and pCDNA3-GATA1 WT or variant vectors as indicated. *: $p<0.01$ vs. GATA1 WT (one-way ANOVA corrected for multiple comparisons). Each plasmid was assayed in five separate transfection experiments. 
1 Supporting information.

\section{Methods}

3 DNA sequencing, variant calling, variant confirmation and segregation studies

4 DNA extraction, next-generation sequencing library preparation, variant calling and annotation were

5 performed as previously described [1]. Briefly, the DNA samples were enriched for the exons of 308

6 genes involved in platelet function and disorders (list available upon request) using HaloPlex

7 technology (Agilent technologies) or SeqCap EZ Choice technology (Roche sequencing). DNA

8 sequencing was then performed on a NextSeq 500 sequencer (Illumina) using 2-channel SBS

9 technology and a Mid-Output kit. Sequence quality was verified using Sequencing Analysis Viewer

11 tool with maximal exact matches (hg19 version of the genome). Variant calling was performed using

12 GATK v3.3.0 and annotated with ANNOVAR [2] and VarAFT software [3]. We applied the following

17 Structural modeling

18 Structural modeling of the GATA1 (NP_002040.1) variants was performed using molecular

19 visualization and modeling with Coot and PyMOL software (The PyMOL Molecular Graphics System;

20 Version 1.5.0.4; Schrödinger LLC).

\section{Flow cytometry analysis}

22 Major platelet receptors and granule markers were assessed as previously described [18]. Briefly, 23 PRP samples were incubated either with or without TRAP-14 (Polypeptide group) and antibodies 24 against $\alpha \mathrm{llb} \beta 3$ integrin (clone P2, Beckman Coulter), active form of $\alpha$ llb $\beta 3$ (clone PAC-1; BD), GPIba 25 (clone HIP1, BD Biosciences), CD63 (clone CLB-gran 12; Beckman Coulter) and CD62P (clone CLB- 
1 Thromb/6, BD Biosciences) for $30 \mathrm{~min}$ at room temperature. Scatter signals and fluorescence

2 intensity were analyzed using an FC500 Flow Cytometer (Beckman Coulter). Circulating CD34+ cells

3 were quantified as previously reported [27].

\section{Cell expression and luciferase reporter assays}

5 Wild-type GATA1 cDNA (amplified from pPacGATA1, a gift from Jeffrey W. Taub) [7] was inserted 6 into a pCDNA3.1-cMyc expression vector. The GATA1 variants were generated using a GENEART 7 Site-directed Mutagenesis System (Life technologies) according to the manufacturer's protocol. The 8 primer sequences used are available upon request. The MYH10 regulatory regions (intron 8 and the 9 3' untranslated region [UTR]) were cloned into a pGL3 vector (Proteogenix). Polymerase chain 10 reaction mutagenesis was used to substitute the GATA1 binding sites with an AAAA sequence. The 11 plasmids were denoted M1/M2/M3/M4 when the four binding sites were substituted and M1, M2, M3 12 or M4 when a single binding site was substituted. After cloning and transformation, each genotype 13 was confirmed using Sanger DNA sequencing (Beckman Coulter Genomics). To examine cell-type 14 dependence, we used the HEL hematopoietic and GripTite 293 MSR (HEK 293 cell line expressing 15 the human macrophage scavenger captor) cell lines. The cells were transfected with pCDNA3.116 cMyc-GATA1 along with the SV40 drive luciferase plasmid (pGL473-hRLuc) to normalize for 17 transfection efficiency. Polyjet (Tebu-bio) was used for transfection according to the manufacturer's protocol. The cells were harvested and lysed 48 hours after transfection. Firefly and Renilla luciferase 19 activity were then measured consecutively using a Dual-Luciferase Reporter 1000 Assay system 20 (Promega) with an EnSight ${ }^{\mathrm{TM}}$ Multimode Microplate Reader (PerkinElmer) according to the 21 manufacturer instructions.

$22 \quad$ PRP serotonin level

23 PRP was prepared according to standard procedures and then frozen at $-70{ }^{\circ} \mathrm{C}$. After thawing and 24 vortexing the sample, the serotonin levels were assessed using high performance liquid 25 chromatography with the following parameters: isocratic flow $0.95 \mathrm{ml} /$ minute, sensor potential $0.5 \mathrm{~V}$, 26 sensitivity $10 \mathrm{nA}$, column temperature $30 \pm 1^{\circ} \mathrm{C}$, analysis time 10 minutes, injection volume $20 \mu \mathrm{l}$, 27 serotonin retention time 5.2 minutes, eluent retention time 6.8 minutes. The serotonin levels were 
1 then expressed according to the PRP platelet count. The plasma serotonin levels were considered

2 negligible as compared with the intraplatelet serotonin content.

\section{Platelet PA1-1 antigen level}

4 Serum was prepared from blood collected in glass tubes and incubated at $37^{\circ} \mathrm{C}$ for 120 minutes

5 before centrifugation at $2000 \mathrm{~g}$ for 10 minutes at $4^{\circ} \mathrm{C}$. The supernatant was stored at $-70^{\circ} \mathrm{C}$ until

6 downstream assays were performed. PAI-1 antigen concentrations in plasma and serum samples

7 were assessed using an enzyme-linked immunosorbent assay (Asserachrom, Diagnostica Stago).

8 The serum results are expressed according to the PRP platelet count after subtracting the plasma

9 values.

10 Mepacrine uptake and release assay

11 PRP samples were prepared according to standard procedures and diluted to obtain a platelet 12 concentration of $10^{7}$ platelets $/ \mathrm{mL}$. The diluted PRP samples were incubated with $1 \mu \mathrm{M}$ or $2 \mu \mathrm{M}$ 13 mepacrine (Q3251, Sigma-Aldrich) for $30 \mathrm{~min}$ at $37^{\circ} \mathrm{C}$; diluted control PRP samples were incubated 14 without mepacrine. Next, the platelet samples were incubated either with or without $40 \mu \mathrm{M} \mathrm{TRAP}-14$. 15 Platelet-mepacrine fluorescence was analyzed using a Navios Cytometer (Beckman Coulter). 16 Mepacrine-labeled dense granules were also quantified via fluorescence microscopy. In single-plane 17 views, mepacrine displayed characteristic punctate staining. A total of 100 platelets were examined, 18 and the number of dense granules per platelet was determined.

\section{Epifluorescence microscopy}

20 Forty-eight hours after transfection, H9C2 cells (ATCC CRL-1446) were fixed in 4\% 21 paraformaldehyde for 20 min at room temperature. The cells were then washed, permeabilized with $220.3 \%$ Triton X100 in PBS for 5 min, blocked with 3\% BSA PBS, and incubated overnight with rabbit 23 anti-cMyc antibody (Santa Cruz Biotechnology; sc-789). Next, the cells were incubated with anti24 rabbit Alexa-546-labeled secondary antibody (Life technologies A11010), and DAPI staining was 25 performed. Finally, after washing the cells, the slides were mounted with Fluoromount and examined 26 using an AXIO Imager M1 microscope (Carl Zeiss, Germany).

27 


\section{Platelet lysates, FOG1/GATA1 immunoprecipitation and western blot analysis}

2 The platelet lysates were prepared as previously described.[8] GripTite 293 MSR cells were co-

3 transfected with the vectors pFN21A-halotag-FOG1 (a gift from Dr. Fujiwara)[9] and pCDNA3-cMyc

4 GATA1. Transfected MSR cells were treated with DTBP reagent (Interchim) for crosslinking and lysed

5 with radio-immunoprecipitation assay (RIPA) buffer (Sigma-Aldrich) supplemented with EDTA-free

6 protease cocktail inhibitor (Roche). The immunoprecipitation was carried out overnight at $4^{\circ} \mathrm{C}$ by

7 incubating the cell extracts with rabbit anti-cMyc coated with magnetic beads (PureProteome,

8 Millipore). After washing the beads with lysis buffer, the immunoprecipitates were suspended in

9 sample loading buffer (NUPAGE LDS sample buffer, Thermo Fisher Scientific) and boiled for $5 \mathrm{~min}$

10 at $95^{\circ} \mathrm{C}$. The immunoprecipitated proteins were assessed for halotag-FOG1 via western blot.

11 The proteins were separated on NUPAGE gels with MES SDS running buffer (Thermo Fisher

12 Scientific) and then transferred onto a polyvinylidene fluoride membrane. The membranes were

13 blocked (5\% Blotto buffer) and labeled overnight with the following primary antibodies: rabbit anti-

14 MYH10 (Cell Signaling Technology), anti-GATA1 (Thermo Fisher Scientific), mouse anti-halotag

15 (Promega), anti-GPVI, anti-SYK, anti-PLC2, anti-COX, anti-TBXAS, anti-cMyc (Santa-Cruz

16 Biotechnology) and anti-GAPDH (Millipore). The membranes were then incubated with the

17 appropriate horseradish peroxidase-conjugated secondary antibody (Bio-Rad Laboratories). Staining

18 was performed using ECL detection reagent (Life Technologies), and the chemiluminescent blots

19 were imaged using the charge-coupled device camera system ImageQuant LAS 4000 (GE

20 Healthcare) and quantified using ImageJ software (National Institutes of Health, Bethesda, MD, USA). 


\section{Supplemental figures}
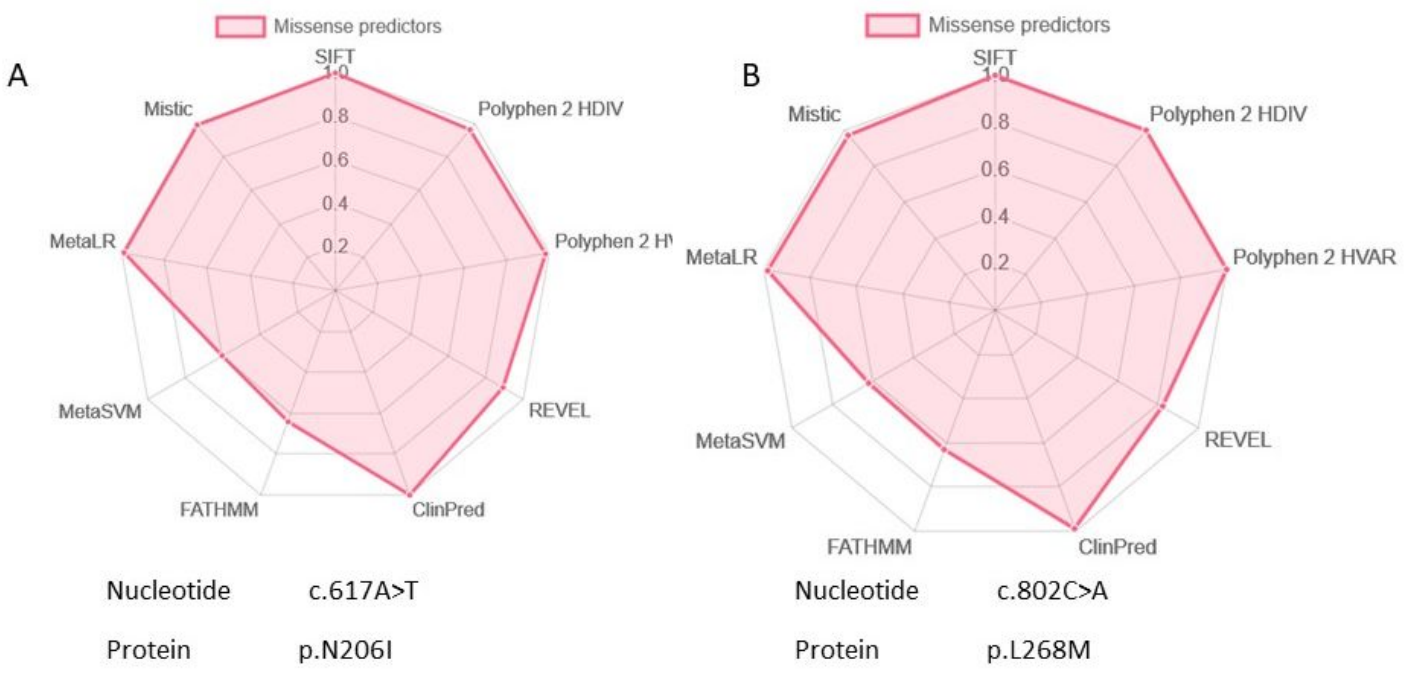

C

\section{Human GATA1 protein and identifiedvariants}

Asn206Ile

PLP PCEARE CVNCGATAT PLWRRDRTGHYLCNACGLYHKMNG ONRPLIRPKKRLIVSKRAGT CTNCQTTTTTLWRRN PLPPCEARE CVICGATAT PLWRRDRTGHYLCNAC FLYHKMNGQNRPL IRPKKRLIVSKRAGT CCTNCQTTTTTLWRRN

Leu268Met PLP PCEARECVNCGATAT PLWRRDRTGHYLCNA OGLYHKMNGONRPLIRPKKRL IVSKRAGT CCTNCQTTTTIMWRRN

\section{Orthologpus protens}

Mouse

Rat

Chicken

Zebrafish

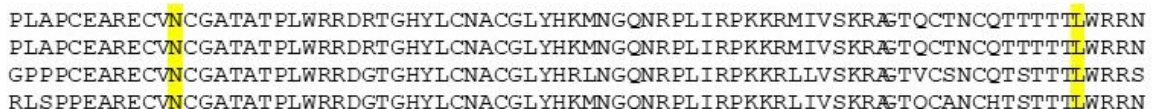

GATA family

GATA1

GATA2

GATA3

GATA4

GATA5

PLPPCEARECVNCGATAT PLWRRDRTGHYLCNACGLYHKMNGQNRPLIRPKKRLIVSKRAGTQCTNCQTTTTHWRR ARSCSEGRECVNCGATAT PLWRRDGTGHYLCNACGLYHKMNGQNRPLIKPKRRLSAARRAGTCCANCQTTTTILWRR ARS-STGRECVNCGATST PLWRRDGTGHYLCNACGLYHKMNGONRPLIKPKRRLSAARRAGTSCANCQTTTTILWR DD-FSEGRECVNCGAMST PLWRRDGT GHYLCNACGLYHKMNGINRPLIKPQRRLSASRRVGLSCANCQTTTTTWRR EEFPGEGRECVNCGALST PLWRRDGT GHYLCNACGLYHKMNGWRPLVRPQKRLSSSRRAGLCCTNCHTTNTIIWRR ED-LSESRECVNCGS IQTPLWRRDGTGHYLCNACGLYSKMNGISRPLIKPQKRVPSSRRLGLSCANCHTTTTIWRR

3 Supplemental Figure 1: Radar view of missense predictors and GATA alignments.

4 (A-B) Radar view of missense predictors (issued from https://mobidetails.iurc.montp.inserm.fr/MD/). $5 \quad$ Values are normalized (0-1) 0 being the less damaging and 1 the most for each predictor algorithms.

6 (C) The variants reported in this study are indicated in red (top alignment). Alignments of GATA1 7 orthologous and different members of the GATA family are provided (middle and bottom alignments, 8 respectively). The boxes delineate both zinc finger domains, ZF1 (position 204-228) and ZF2 (position 9 258-282). The GATA1 reference variant is NM_002049. WT: wild-type. 


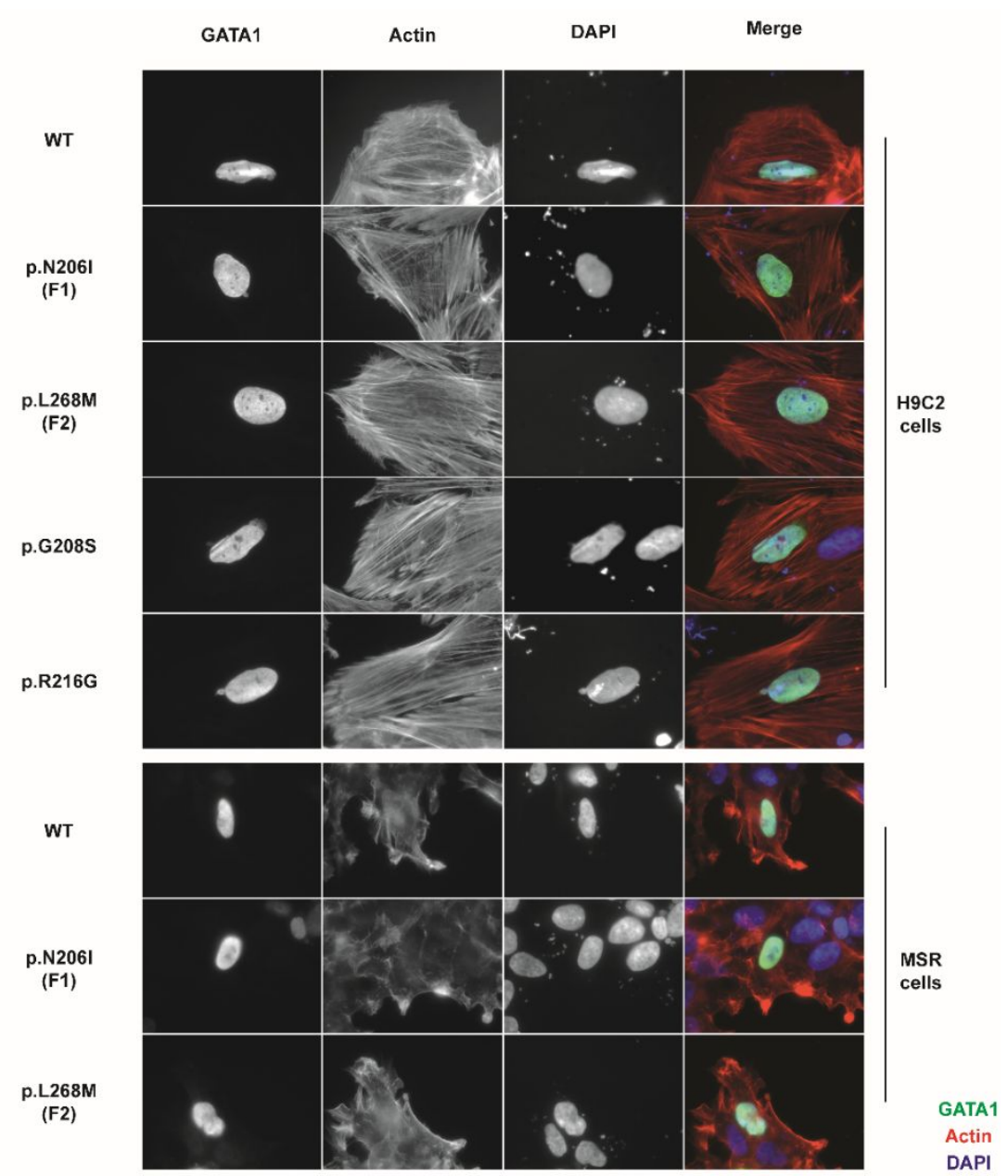

B

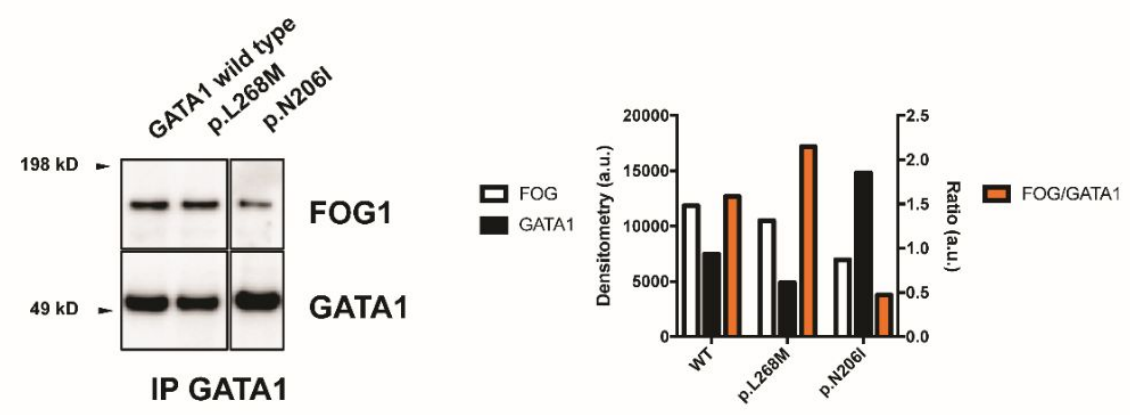

\section{Supplemental Figure 2: Functional characterization of the GATA1 variants.}

(A) Subcellular localization of the GATA1 variants. Representative immunofluorescence microscopy images of H9C2 (upper panel) and MSR (lower panel) cells transfected with wild-type (WT) GATA1 or variants (pCDNA3-cMyc-GATA1 expression vectors) after cMyc, actin and DAPI staining (100X magnification). The p.N206I and p.L268M variants were compared to the previously reported variants p.G208S [10] and p.R216G [11,12] as well as WT GATA1.

(B) Immunoprecipitation of GATA1-cMyc in GripTite 293 MSR cells co-transfected with pFN21AHaloTag-FOG1 and pCDNA3-cMyc GATA1 (wild-type or variants) vectors. Immunoprecipitation was performed on cell lysate with rabbit anti-cMyc antibody (Santa-Cruz, sc-789). The immunoprecipitates 
1 were analyzed via western blot using murine anti-HaloTag (Promega, G921A) and anti-cMyc (Santa2 Cruz, sc-40) antibodies. Input correspond to the GATA1 and FOG1 levels before IP. All samples were 3 run together and the bands were detected simultaneously (the original photograph can be provided 4 on request). Quantification of band intensity for FOG1, GATA1 and ratio GOG1/GATA1 is shown to 5 the right of the western blot. 
A

F2-I-1
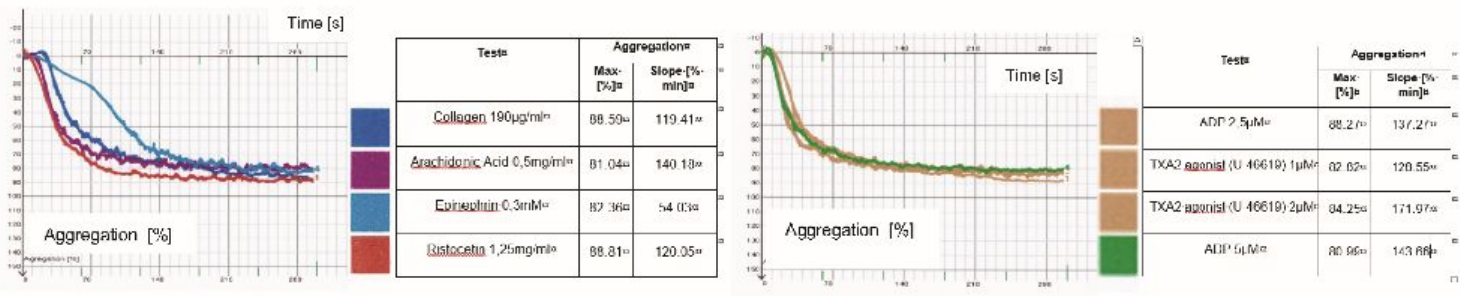

B

F2-I-2

C

F2-II-1
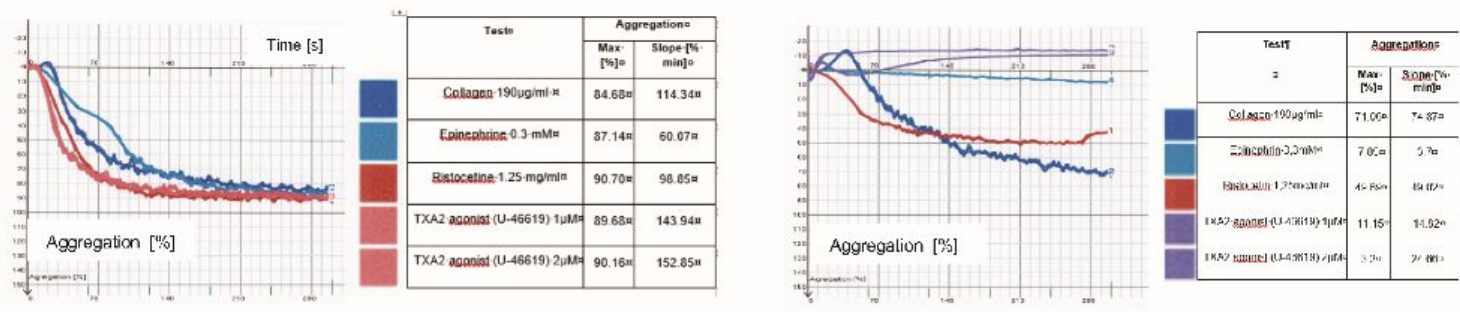

F2-II-2

D

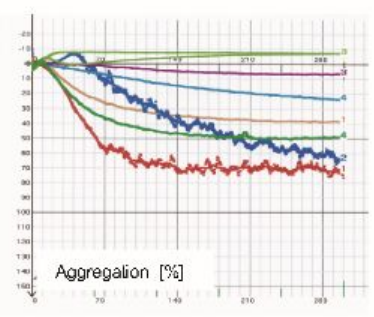

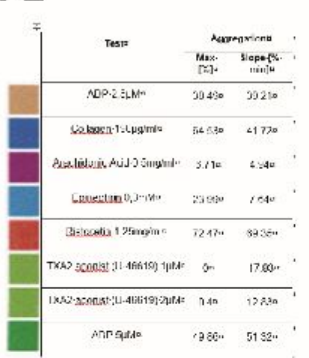

E F2-I-1, F2-II-1 and F2-II-2

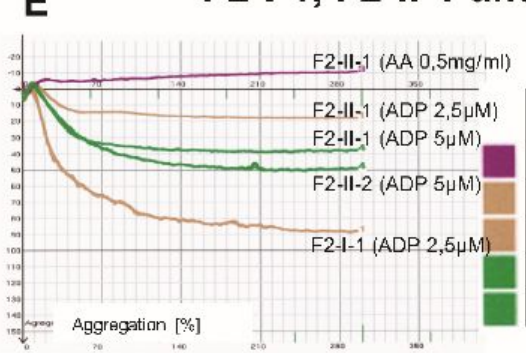

\begin{tabular}{|c|c|c|}
\hline \multirow{2}{*}{ Test } & \multicolumn{2}{|c|}{ Aopreastions } \\
\hline & $\prod_{|\rightarrow| x \mid}$ & 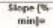 \\
\hline 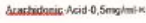 & -5710 & -0.654 \\
\hline$A D P=2,5 \mathrm{y} M \mathrm{M}$ & 88.278 & 137278 \\
\hline$A D P-2,5 \mathrm{SP} M \mathrm{~m}=$ & 18.135 & 38978 \\
\hline ADP 5 SUMO & $49.86=$ & 51.324 \\
\hline ADP SUASAM & 30.480 & 02 45E \\
\hline
\end{tabular}

$\mathbf{F}$

F2-I-1, F2-I-2 and F2-II-2
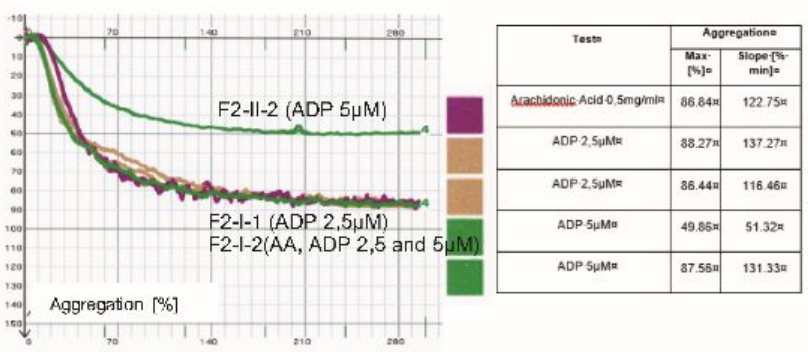

Supplemental Figure 3: Platelet aggregation tracings for F2 family (pL268M).

A-D Aggregation tracings for F2-I-1 (mother), F2-I-2 (father), F2-II-1 (firstborn son) and F2-II-2 (second son) family members, on separated graphs. E- Aggregation tracings for F2-I-1, F2-II-1 and F2-II-2 on the same graphs. F- Aggregation tracings for F2-I-1, F2-I-2 and F2-II-2 on the same graphs. Platelet aggregation was induced by different agonist and different agonist concentrations indicated in the table, on the right to the curves. Percentage max of aggregation and slope were indicated in the table. 
1

2

3

4

5

6

7

8

9

10

11

12

13

14

15

16

17

18

19

20

21

22

23

24

25

26

27

28

29
A
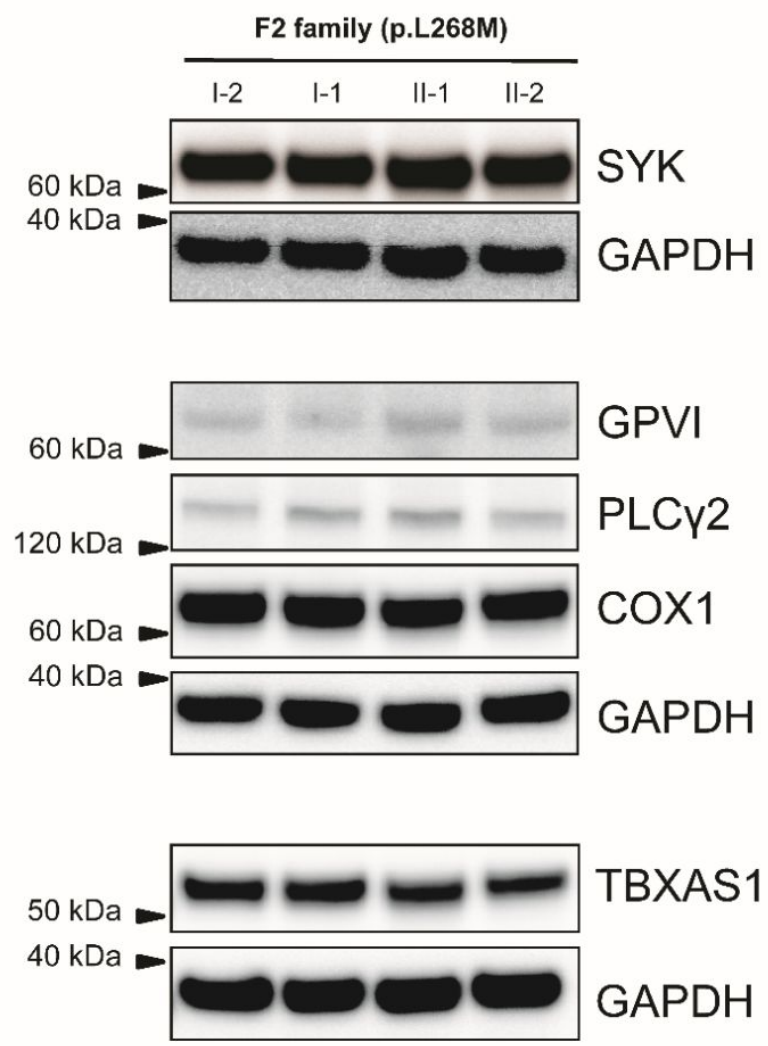

B

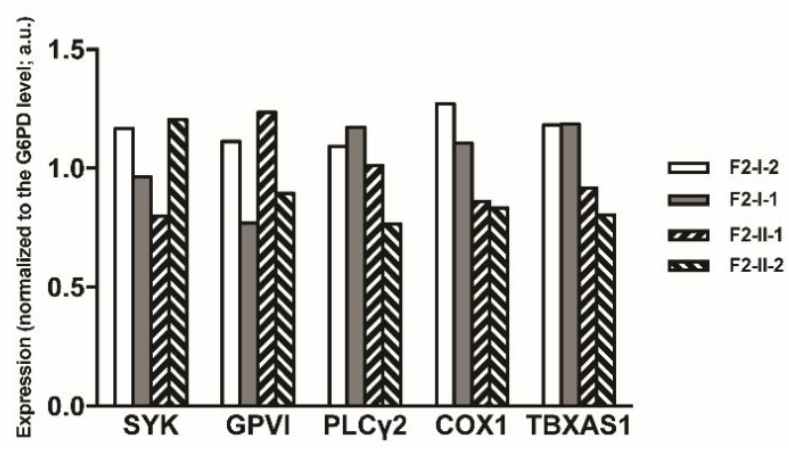

\section{Supplemental}

Figure 4: Evaluation of proteins involved in the collagen and arachidonic acid signaling pathways

Expression of the proteins involved in the collagen (GPVI, SYK and PLCY2) and arachidonic acid (COX, TBXAS1) signaling pathways were analyzed via western blot of platelet lysates $(30 \mu \mathrm{g})$ from the F2 family members: the GATA1 variant carriers (F2-II-1 and F2-II-2), maternal heterozygous carrier (F2-1-1) and unaffected father (F2-I-2). Quantification of band intensity is shown below the western blots. 
A

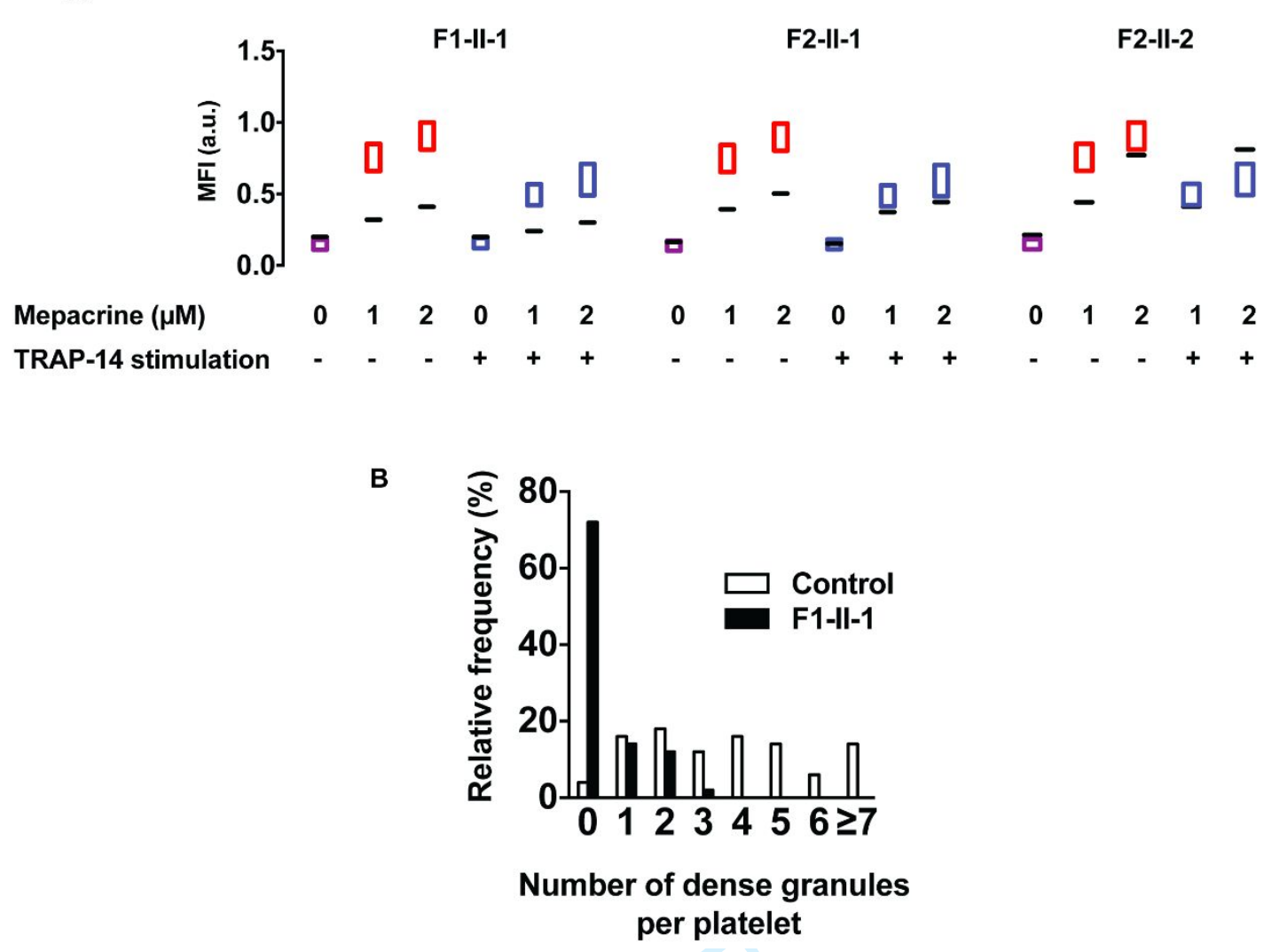

2 Supplemental Figure 5: Mepacrine test

3 (A) Flow cytometric mepacrine uptake and release assays of the three variant carriers and unrelated 4 controls. The platelets were incubated with $1 \mu \mathrm{M}$ or $2 \mu \mathrm{M}$ mepacrine and stimulated with TRAP-14 $5 \quad(50 \mu \mathrm{M})$ to evaluate mepacrine release. Rectangles indicate the reference values, while black 6 horizontal bars indicate the patient values. MFI: mean fluorescence intensity.

7 (B) Quantification of dense granules in 100 platelets using immunofluorescence microscopy after 8 mepacrine uptake in patient F1-II-1 and a control. 
A
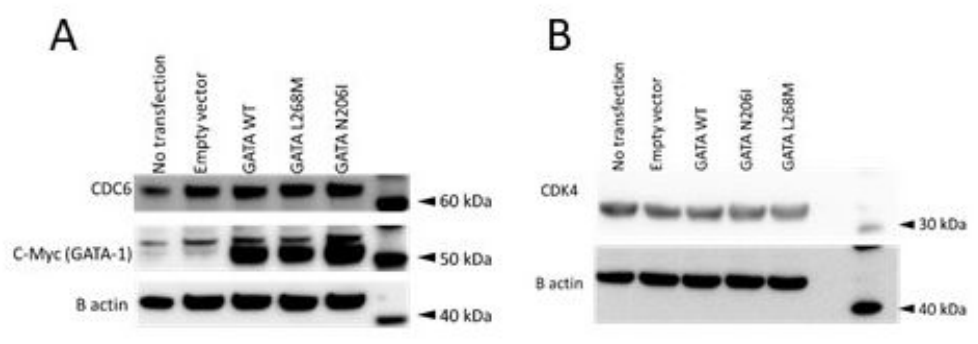

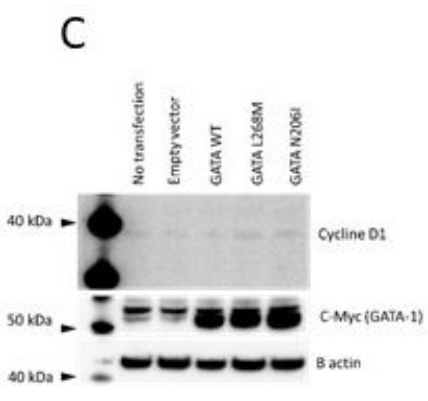

1

2 Supplemental Figure 6: Evaluation of proteins involved in GATA-1 mediated polyploidization 3 defect.

4 Western blot analysis of CDC6 (A), CDK4 (B) and Cyclin-D1 (C) expression in GripTite 293 MSR cell 5 lines transfected with GATA-1 wild-type or variants. GATA-1-cMyc was assessed as a transfection 6 control. Actin expression was assessed as a protein loading control. A and B panels shows the same 7 samples. 


\section{3'UTR sequence hg38_dna range=chr17:8472947-8473157}

CAAAGCCCAGCTGCCATAAACTGTCTTCCTTGTGCTCTGAATGCCCAACGAAGGTTGTATAAACTACC TTTCCTTAGGTCTGGAGTGTCGGGTCAGTCAAGAAGGGACCCTTTTAGTTATTAAATAGTATCTTTGC AGAATCAGGGCCTGGAATTGGTTCACACAGATTGTTTCTCCTGGCACGCTGAGTAGTTGGGAAACTGT GACGTGA

\section{Intronic sequence}

hg38_dna range=chr17:8553047-8553739

\section{Wild-type}

TGGGGCCACCAAAGTAAATTAATTCTGTATGTGTCAGATAGCCTGAATTTTAAGTCAGACTA TCCCACACATCCTCCACGTCTGGTGAAAGACACACTCATATGAATCATGCTCTGACACACCT ATATGAAGAAAGCTGAACTGCAGATTCATTCTCCTGTCATTTTCAAGTATTTCCAGATTTTG CTGGCTGTGAGTCCATTTGGAGGCATAAAATACTGTTCTTAACCAGAAAATCATGGTCTGTC CCTTGAAGGATAGAAATAATGTATTTCTATCACTGGAGATTATTCTATTAAGTACCCGAAAA CTGTCATCTATCTTTCTAATTGGCACACTATCATTCAGAATCATTTCTATTTTATTACATCT ATACTCAAATGTGTTTACCTGGGTATATATGTGCATACACATATATAAACCTTCTTATGGC CATTCTATGCAGGTGACATTTCTTAGGACTATTCTATGTACTTGACATTTTATTTTTTTCTC CAGCTTTATTCTACTTAACACTAATTGAGATTACTTTAACTCTGTAATCTTATAAGGATGTT TTCAATGATTACATGCTCATCCTTCTAATATCAGAAGTAGATGGTAAAAAGTGGGCTAACAG CAATAGGGGGAGGGCAACTGCATTCATCTGAATGTCCTATATCACACAAACACTGTGGCACT СTCСACTTCCC pGL3 intron 8 MYH10 WT

\begin{tabular}{|lllll|}
\hline AGATAG & CTATCA & CTATCT & CTATCA \\
\hline BS-1 & BS-2 & BS-3 & BS-4
\end{tabular}

Mutated binding-site (BS-M1,2,3,4)

TGGGGCCACCAAAGTAAATTAATTCTGTATGTGTCAAAAAGCCTGAATTTTAAGTCAGACTA TCCCACACATCCTCCACGTCTGGTGAAAGACACACTCATATGAATCATGCTCTGACACACCT ATATGAAGAAAGCTGAACTGCAGATTCATTCTCCTGTCATTTTCAAGTATTTCCAGATTTT CTGGCTGTGAGTCCATTTGGAGGCATAAAATACTGTTCTTAACCAGAAAATCATGGTCTGTC CCTTGAAGGATAGAAATAATGTATTTCAAAAACTGGAGATTATTCTATTAAGTACCCGAAAA CTGTCATCAAAATTTCTAATTGGCACACAAAAATTCAGAATCATTTCTATTTTATTACATCT ATACTCAAATGTGTTTACCTGGGTATATATGTGCATACACATATATAAACCTTCTTATGGC CATTCTATGCAGGTGACATTTCTTAGGACTATTCTATGTACTTGACATTTTATTTTTTTCTC CAGCTTTATTCTACTTAACACTAATTGAGATTACTTTAACTCTGTAATCTTATAAGGATGTT TTCAATGATTACATGCTCATCCTTCTAATATCAGAAGTAGATGGTAAAAAGTGGGCTAACAG CAATAGGGGGAGGGCAACTGCATTCATCTGAATGTCCTATATCACACAAACACTGTGGCACT СTCСACTTCCC

\begin{tabular}{|cccc|}
\hline pGL3 intron 8 MYH10 with mutated binding-site \\
\hline BSAAAG & CAAAAA & CAAAAT & CAAAAA \\
\hline BS-M1 & BS-M2 & BS-M3 & BS-M4
\end{tabular}

\footnotetext{
1 Supplemental Figure 7: DNA sequence of regulatory GATA1 binding regions of the 2 MYH10 gene.

3 The sequences of the 3'UTR (Chr. 17: 8472947-8473157) and intronic region (Chr. 17: 8553047-

4 8553739) of the MYH10 gene. The putative consensus binding sites for GATA1 within intron 8 of the

$5 \mathrm{MYH10}$ gene are displayed in italic and underlined in green. Both fragments were cloned into a 6 luciferase construct, 5' of the luciferase reporter gene, to generate pGL3-3'UTR MYH10 WT and 7 pGL3-Intron 8 MYH10 WT. The binding-site mutant constructs are pGL3-Intron 8 MYH10 M1, pGL38 Intron 8 MYH10 M2, pGL3-Intron 8 MYH10 M3, pGL3-Intron 8 MYH10 M4 and pGL3-Intron 8 MYH10 $9 \mathrm{M}(1,2,3,4)$ with the indicated mutations.
} 

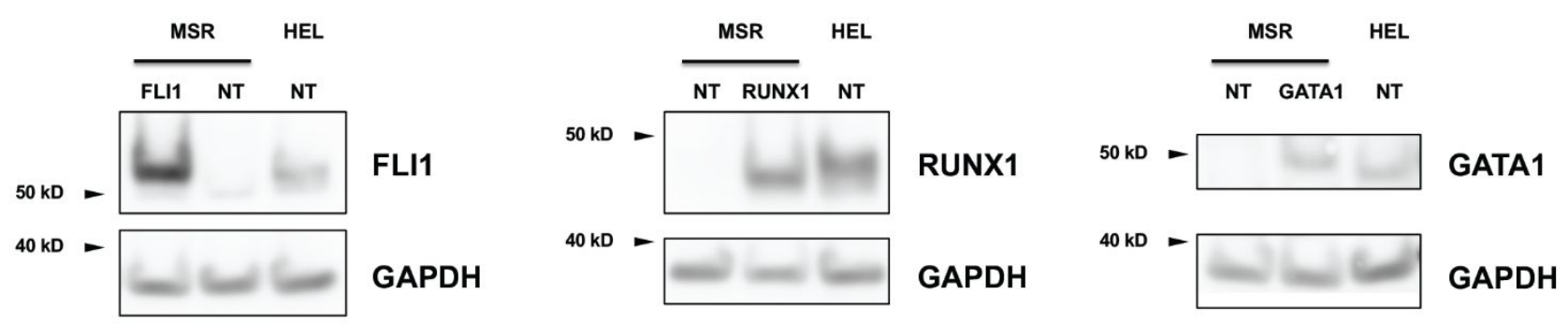

2

3 Supplemental Figure 8: FLI1, RUNX1 and GATA1 transcription factor cell expression

4 Western blot analysis of FLI1, RUNX1 and GATA1 expression in native (non-transfected, NT) HEL

5 cells, native GripTite 293 MSR cell lines, and transfected (with RUNX1, FLI1 or GATA1) GripTite 293

6 MSR cell lines. GAPDH expression was assessed as a protein loading control. 


\section{Supplemental Figure 9: Functional analysis of the MYH10 regulatory regions.}

4 Luciferase expression after transfection in GripTite 293 MSR cells with pGL3-intron 8 MYH10 WT 5 and pCDNA3-GATA1 WT or variant vectors as indicated. ${ }^{* *} p<0.01$ vs. GATA1 WT (one-way ANOVA 6 corrected for multiple comparisons). Each plasmid was assayed in duplicate in one or two separate 7 transfection experiments (pink or black symbols). 
3

4

5

6

7

8

9

10

11

12

13

14

15

16

17

18

19

20

21

22

23

24

25

26

27

28

29

30

31

32

33

34

35

36

37

38

39

40

41

42

43

44

45

46

47

48

49

50

51

52

53

54

55

56

57

58

59

60

\section{Supplemental Table 1 Candidate genes}

2

3

4

\begin{tabular}{|c|c|c|c|c|c|c|c|c|c|c|}
\hline Gene & Variation (NM) & $\begin{array}{c}\text { gnomAD } \\
\text { exome } \\
\text { (v2.0.1 } \\
\text { Exomes } \\
\text { global } \\
\text { MAF) }\end{array}$ & $\begin{array}{l}\text { gnomAD } \\
\text { genome } \\
\text { (Genomes } \\
\text { global } \\
\text { MAF) }\end{array}$ & $\begin{array}{l}\text { gnomAD } \\
\text { (v3 } \\
\text { Genomes } \\
\text { global } \\
\text { MAF) }\end{array}$ & $\begin{array}{l}\text { dbSNP rsid } \\
\text { (Identifier for } \\
\text { NCBI dbSNP) }\end{array}$ & $\begin{array}{c}\text { Clinvar } \\
\text { interpretati } \\
\text { on } \\
\text { (v2021012 } \\
3 \text { ) }\end{array}$ & $\begin{array}{c}\text { CADD } \\
\text { raw: } \\
\text { [- } \\
6.41 ; 35.5] \\
\text { The higher } \\
\text { the less } \\
\text { likely to } \\
\text { be } \\
\text { observed }\end{array}$ & $\begin{array}{c}\text { CADD } \\
\text { phre } \\
\text { d } \\
\text { Phre } \\
\text { d-like } \\
\text { scali } \\
\text { ng of } \\
\text { raw } \\
\text { scor } \\
\text { e } \\
\end{array}$ & $\begin{array}{c}\text { MPA } \\
\text { score } \\
\text { Raw } \\
\text { score } \\
{[0 ; 10] \text {, }} \\
10: \\
\text { high } \\
\text { impac } \\
t\end{array}$ & $\begin{array}{c}\text { MPA } \\
\text { impact }\end{array}$ \\
\hline WDR66 & $\begin{array}{c}\text { C.1915C>T } \\
\left(N_{-144668.5}\right)\end{array}$ & 0.0001 & $9.686 \mathrm{e}-05$ & $8.373 e-05$ & rs202238684 & No match & 6.3 & 35 & 10 & Nonsense \\
\hline LYST & $\begin{array}{l}\text { C.11268-5del } \\
(\mathrm{NM} \text {-000081.3)( }\end{array}$ & 0.5087 & 0.5810 & 0.5679 & No match & $\begin{array}{l}\text { Benign / } \\
\text { Likely } \\
\text { benign }\end{array}$ & 0.079599 & $\begin{array}{c}1.91 \\
5\end{array}$ & 0 & Unknown \\
\hline GCKR & $\begin{array}{c}\text { c.217-8C>T } \\
\left(\mathrm{NM} \_001486.3\right)\end{array}$ & 0.0001 & No match & $2.791 \mathrm{e}-05$ & rs755995385 & No match & 0.26 & 3.88 & 0 & Unknown \\
\hline CD226 & $\begin{array}{c}\text { c.-33delT } \\
\left(\mathrm{NM} \_001303618.1\right)\end{array}$ & 0.0117 & 0.0089 & 0.0091 & rs3214811 & No match & 0.017666 & $\begin{array}{c}1.45 \\
0\end{array}$ & 0 & Unknown \\
\hline RNF145 & $\begin{array}{c}\text { c.1270-9_1270- } \\
\text { 4del } \\
\text { (NM_001199383.1) }\end{array}$ & 0.0015 & 0.0014 & 0.0017 & rs59997682 & No match & 0.704591 & $\begin{array}{c}8.49 \\
7\end{array}$ & 0 & Unknown \\
\hline
\end{tabular}


1 Supplemental Table 2 Morphometric parameters from sections of platelet electron 2 microscopy.

3 See the Methods section for the comparison between the patient and the controls

4

\begin{tabular}{cccccc}
\hline & $\begin{array}{c}\text { Platelet area } \\
\left(\boldsymbol{\mu m ^ { 2 } )}\right.\end{array}$ & $\begin{array}{c}\text { Major platelet } \\
\text { diameter }(\boldsymbol{\mu m})\end{array}$ & $\begin{array}{c}\text { Minor platelet } \\
\text { diameter }(\boldsymbol{\mu m})\end{array}$ & $\begin{array}{c}\text { Alpha granule } \\
\text { diameter }(\mathrm{nm})\end{array}$ & $\begin{array}{c}\text { Alpha granule } \\
\text { per } \mu \mathrm{m}^{2}\end{array}$ \\
\hline Controls $(\mathrm{n}=10)$ & $2.79 \pm 1.60$ & $2.85 \pm 1.23$ & $1.25 \pm 1.49$ & $235 \pm 1.2$ & $2.51 \pm 1.29$ \\
F2-II-1 & $2.74 \pm 1.73$ & $2.75 \pm 1.26$ & $1.27 \pm 1.51$ & $208 \pm 1.4$ & $1.08 \pm 0.81$ \\
p-value & 0.751 & 0.104 & 0.663 & $<0.001$ & $<0.001$ \\
\hline
\end{tabular}




\section{Supplemental references}

1 Saultier P, Vidal L, Canault M, Bernot D, Falaise C, Pouymayou C, Bordet J-C, Saut N, Rostan A, Baccini V, Peiretti F, Favier M, Lucca P, Deleuze J-F, Olaso R, Boland A, Morange PE, Gachet C, Malergue $F$, Fauré $S$, et al. Macrothrombocytopenia and dense granule deficiency associated with FLI1 variants: ultrastructural and pathogenic features. Haematologica 2017; 102: 100616.

2 Wang $\mathrm{K}$, Li M, Hakonarson $\mathrm{H}$. ANNOVAR: functional annotation of genetic variants from highthroughput sequencing data. Nucleic Acids Res 2010; 38: e164.

3 Desvignes J-P, Bartoli M, Delague V, Krahn M, Miltgen M, Béroud C, Salgado D. VarAFT: a variant annotation and filtration system for human next generation sequencing data. Nucleic Acids Res 2018; 46: W545-53.

4 Frédéric MY, Lalande M, Boileau C, Hamroun D, Claustres M, Béroud C, Collod-Béroud G. UMD-predictor, a new prediction tool for nucleotide substitution pathogenicity -- application to four genes: FBN1, FBN2, TGFBR1, and TGFBR2. Hum Mutat 2009; 30: 952-9.

5 Choi Y, Sims GE, Murphy S, Miller JR, Chan AP. Predicting the functional effect of amino acid substitutions and indels. PLOS ONE 2012; 7: e46688.

6 Adzhubei IA, Schmidt S, Peshkin L, Ramensky VE, Gerasimova A, Bork P, Kondrashov AS, Sunyaev SR. A method and server for predicting damaging missense mutations. Nat Methods 2010; 7: 248-9.

7 Caldwell JT, Edwards H, Dombkowski AA, Buck SA, Matherly LH, Ge Y, Taub JW. Overexpression of GATA1 confers resistance to chemotherapy in acute megakaryocytic Leukemia. PLOS ONE 2013; 8: e68601.

8 Poggi M, Canault M, Favier M, Turro E, Saultier P, Ghalloussi D, Baccini V, Vidal L, Mezzapesa A, Chelghoum N, Mohand-Oumoussa B, Falaise C, Favier R, Ouwehand WH, Fiore M, Peiretti F, Morange PE, Saut N, Bernot D, Greinacher A, et al. Germline variants in ETV6 underlie reduced platelet formation, platelet dysfunction and increased levels of circulating CD34+ progenitors. Haematologica 2017; 102: 282-94.

9 Fujiwara T, Sasaki K, Saito K, Hatta S, Ichikawa S, Kobayashi M, Okitsu Y, Fukuhara N, Onishi Y, Harigae H. Forced FOG1 expression in erythroleukemia cells: Induction of erythroid genes and repression of myelo-lymphoid transcription factor PU.1. Biochem Biophys Res Commun 2017; 485: 380-7.

10 Mehaffey MG, Newton AL, Gandhi MJ, Crossley M, Drachman JG. X-linked thrombocytopenia caused by a novel mutation of GATA-1. Blood 2001; 98: 2681-8.

11 Yu C, Niakan KK, Matsushita M, Stamatoyannopoulos G, Orkin SH, Raskind WH. X-linked thrombocytopenia with thalassemia from a mutation in the amino finger of GATA-1 affecting DNA binding rather than FOG-1 interaction. Blood 2002; 100: 2040-5. 
12 Balduini CL, Pecci A, Loffredo G, Izzo P, Noris P, Grosso M, Bergamaschi G, Rosti V, Magrini U,

$\begin{array}{ll}2 & \text { Ceresa IF, Conti V, Poggi V, Savoia A. Effects of the R216Q mutation of GATA-1 on } \\ 3 & \text { erythropoiesis and megakaryocytopoiesis. Thromb Haemost 2004; 91: 129-40. }\end{array}$

4

5

6 